\title{
Variation in Structure of the Subcanopy Assemblage Associated with Southern California Populations of the Intertidal Rockweed Silvetia compressa (Fucales) ${ }^{1}$
}

\author{
Stepbanie A. Sapper ${ }^{2}$ and Steven N. Murray ${ }^{2,3}$
}

\begin{abstract}
Variation in structure of the subcanopy communities associated with southern California Silvetia compressa (J. Agardh) Serrão, Cho, Boo \& Brawley populations was examined at eight sites, including four long-standing intertidal Marine Protected Areas (MPAs). Although sea temperature and salinity showed little variation, maximum wave force and sand influence differed significantly among sites. Seaweed and sessile macroinvertebrate cover and mobile macroinvertebrate densities were determined in 10 quadrats during both autumn 1995 and spring 1996. A total of 111 taxa was distinguished at the eight sites, including 47 macroalgae, 20 sessile macroinvertebrates, and 44 mobile macroinvertebrates; however, only a few species consistently dominated abundances in the subcanopy assemblage. Silvetia compressa cover varied significantly among sites during both sampling periods; cover was significantly greater at all but one site during the autumn. Morphologies of Silvetia compressa thalli were qualitatively similar except at Monarch Bay, where plants were the least densely aggregated and frond lengths were two to three times greater than at other sites. Seaweeds contributed $71.2 \%$ of the subcanopy cover averaged over all sites compared with $23.8 \%$ sessile macroinvertebrate cover; mobile invertebrate densities averaged $363.9 \mathrm{~m}^{-2}$ over all sites. The three most abundant seaweeds (Pseudolitboderma nigra, Pseudolitbophyllum neofarlowi, and Corallina pinnatifolial C. vancouveriensis) and macroinvertebrates (Phragmatopoma californica, Mytilus californianus, and Antbopleura elegantissima) accounted for approximately $67 \%$ and $20 \%$, respectively, of total understory cover. The three most abundant mobile macroinvertebrates (Littorina scutulata, Lepidochitona hartwegii, and Macclintockia scabra/Lottia conus) accounted for nearly $60 \%$ of all mobile animals. An average of 27 macrophytes and sessile macroinvertebrates and 19 mobile macroinvertebrates occurred at a site; site $H^{\prime}$ diversity based on macrophyte and sessile macroinvertebrate cover averaged 1.91 ; mobile macroinvertebrate $H^{\prime}$ diversity based on density averaged 2.03. Neither cluster analysis nor multidimensional scaling produced clear site patterns based on geographic location or sampling period; long-standing MPA sites did not form a distinct group and did not differ significantly in community structure from nonhistorical MPAs based on Analysis of Similarity (ANOSIM) tests. Communities representing autumn and spring were more closely associated with each other than with communities from other sites. Differences in community structure were detected among individual sites in all ANOSIM tests despite strong similarities in abundant taxa. ANOSIM tests also showed that understory communities differed between
\end{abstract}

1 Funded by National Sea Grant College Program, NOAA, U.S. Department of Commerce under Grant NA

Pacific Science (2003), vol. 57, no. 4:433-462

(C) 2003 by University of Hawai' $i$ Press

All rights reserved
46 RG 0472, the California Resources Agency, and Minerals Management Service (Pacific OCS). Manuscript accepted 18 March 2003.

${ }^{2}$ Department of Biological Science, California State University, Fullerton, Fullerton, California 92834-6850 (phone: 714-278-7291; fax 714-278-7095; E-mail: smurray@fullerton.edu).

${ }^{3}$ Corresponding author. 
sampling periods, except for analyses based on cover in recently established MPAs. Significant differences in the cover and density of many abundant subcanopy populations also were found among sites using univariate statistical procedures. Only weak relationships could be established between variations in species types and environmental factors. These results suggest the importance of localized and stochastic histories in generating site variation among rockweedassociated populations and the difficulties in establishing post hoc relationships between environmental patterns and variations in species abundances.

VARIATIONS IN ROCKY intertidal communities at the scale of the individual site have received much attention. For example, the vertical distributions and abundances of species populations as a function of tidal height (e.g., Lewis 1964, Stephenson and Stephenson 1972, Ricketts et al. 1985) and the temporal dynamics of patch formation and colonization (reviewed by Paine and Levin 1981, Sousa 1985) are well known. In addition, field assessments of control and impacted study areas or sites, separated by distances of meters to several kilometers, have formed the basis for determining the effects of anthropogenic and natural disturbances on rocky intertidal communities (Green 1979, Stewart-Oaten et al. 1986, Osenberg and Schmitt 1996). Few studies, however, have addressed the degree of variation in the structure of communities occupying sites exposed to similar oceanographic conditions and distributed over spatial scales of only a few kilometers.

Fucacean rockweeds are persistent features of semiprotected, temperate rocky intertidal communities, where they form assemblages characterized by a diversity of invertebrates and seaweeds (Lubchenco 1983, Foster et al. 1991b, Mathieson et al. 1991, Thompson et al. 1996). These rockweeds compete for primary space with sessile invertebrates, such as barnacles and mussels (Mann 1982), and protect understory species from desiccation during tidal emersion. Fucoid fronds also contribute to the disturbance of subcanopy organisms through whiplash (Southward 1956, Hawkins and Hartnoll 1983a) and by harboring high densities of invertebrate grazers (Hawkins and Hartnoll 1983b, Lubchenco 1983, Van Alstyne 1990).

Because of their abundance, ecological importance, and restricted vertical distribu- tions on temperate shores (Lewis 1964, Stephenson and Stephenson 1972, Foster et al. 1991b), fucacean communities are excellent candidates for studies of site-scale variation in population and community parameters. Most previous work on fucoid communities has concentrated on factors influencing the distribution and abundance of the canopyforming rockweeds (Chapman 1995), including grazers (Lubchenco 1983, Chapman and Johnson 1990, Menge 1991), wave force (Pielou 1981, Vadas et al. 1990), desiccation (Schonbeck and Norton 1978), and sand inundation (Daly and Mathieson 1977). Few studies have concentrated on the organisms that live beneath these canopies and the degree to which the structure of subcanopy assemblages varies among sites distributed over short distances.

In the North Pacific, most ecological research on rockweed communities has focused on Fucus and its associates. Much less is known about communities dominated by Silvetia compressa (J. Agardh) Serrão, Cho, Boo \& Brawley (formerly known as Pelvetia fastigiata (J. Agardh) De'Toni), a fucacean seaweed that characterizes protected and semiprotected rocky intertidal habitats throughout central and southern California (Abbott and Hollenberg 1976, Ricketts et al. 1985, Murray and Bray 1993). Previous studies have addressed the demography (Gunnill 1980, 1985) and genetic structure (Williams and Di Fiori 1996) of S. compressa populations and the abundances of their microepibiotic faunal associates (Gunnill 1982, 1983, 1984, 1985); to our knowledge, only Hill (1980) has described the macroorganism populations found beneath $S$. compressa fronds.

The purpose of this research was to characterize among-site and temporal variation in the structure of the subcanopy seaweed 
and macroinvertebrate communities associated with southern California Silvetia compressa populations. A second purpose was to determine whether $S$. compressa assemblages in Marine Protected Areas (MPAs) established more than $25 \mathrm{yr}$ ago could be distinguished from those at sites only very recently accorded MPA protection.

\section{MATERIALS AND METHODS}

\section{Study Sites}

Eight sites were established along ca. 15.5 $\mathrm{km}$ of southwest-facing coastline in Orange County, California (Figure 1, Table 1). This coastline consists mostly of a composite of rocky headlands and pocket beaches, backed by eroded bluffs, and is semiprotected from predominant wave patterns by offshore islands (Hickey 1993). All study areas were characterized by conspicuous $S$. compressa assemblages, which occupied largely horizontal, rocky substrata. Study sites were separated by distances ranging from 1.1 to $5.1 \mathrm{~km}$ and showed little site-to-site variation in sea temperature $\left(\leq 1^{\circ} \mathrm{C}\right)$ and salinity $(\leq 1 \%)$. Sea temperature in this region ranged annually throughout the 1980 s from seasonal means of 13 to $21^{\circ} \mathrm{C}$ and salinity from 32 to $34 \%$ (Brophy and Murray 1989). During the study, temperatures near the study site ranged from 13.2 to $19.0^{\circ} \mathrm{C}$ and salinities from 31.6 to $33.6 \%$ (Balboa, Newport Beach, California; ftp://nemo.ucsd.edu/pub/shore). Four of the eight sites were intertidal MPAs (California Marine Life Refuges) where the collection of marine plants and most species of

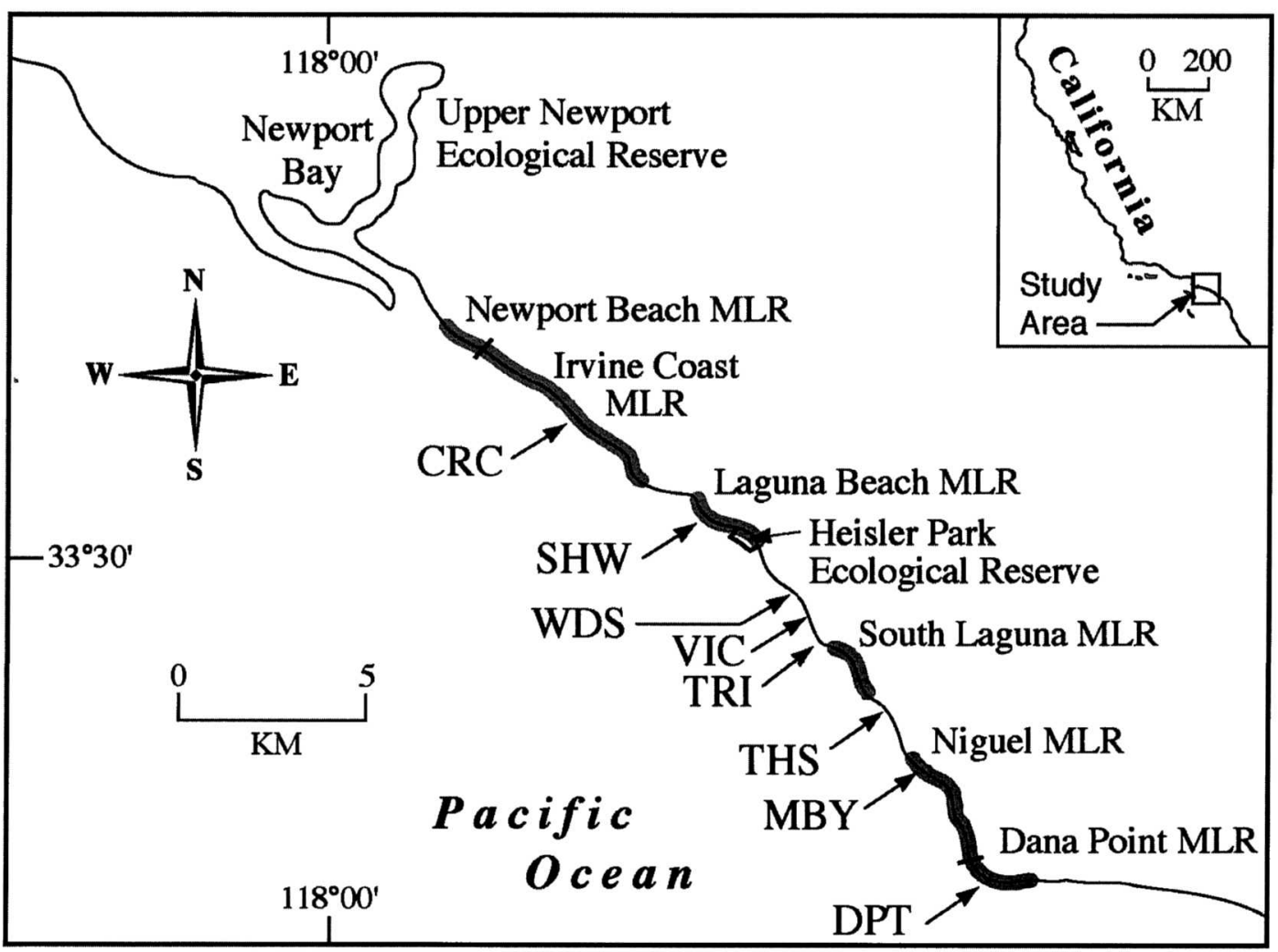

Figure 1. Map of the eight southern California study sites. Study site codes: CRC, Crystal Cove; SHW, Shaw's Cove; WDS, Woods Cove; VIC, Victoria Beach; TRI, Treasure Island; THS, Thousand Steps; MBY, Monarch Bay; DPT, Dana Point. Shaded areas represent boundaries of local MPAs (California Marine Life Refuges and Ecological Reserves) established before 1994 in which collecting of invertebrates is prohibited. 
TABLE 1

Characteristics of the Eight Study Sites, Including Latitude and Longitude, Geological Formation, General Site Topography, and Vertical Tidal Range of the S. compressa Assemblage

\begin{tabular}{|c|c|c|c|c|}
\hline Site ${ }^{a, b}$ (Abbreviation) & $\begin{array}{l}\text { Latitude and } \\
\text { Longitude }\end{array}$ & $\begin{array}{c}\text { Geologic } \\
\text { Formation }{ }^{c}\end{array}$ & Site Topography & $\begin{array}{l}\text { Vertical } \\
\text { Range (m) }\end{array}$ \\
\hline †Crystal Cove (CRC) & $\begin{array}{l}33^{\circ} 34^{\prime} 13^{\prime \prime} \mathrm{N} \\
117^{\circ} 50^{\prime} 15^{\prime \prime} \mathrm{W}\end{array}$ & $\operatorname{Tm}$ & $\begin{array}{l}\text { Flattened and angled benches } \\
\text { separated by crevices resulting } \\
\text { between uplifted bedding planes }\end{array}$ & +0.7 to +1.2 \\
\hline †Shaw's Cove (SHW) & $\begin{array}{l}33^{\circ} 32^{\prime} 42^{\prime \prime} \mathrm{N} \\
117^{\circ} 47^{\prime} 57^{\prime \prime} \mathrm{W}\end{array}$ & Tso & $\begin{array}{l}\text { Flattened and gently sloping benches } \\
\text { separated by crevices and channels }\end{array}$ & +1.0 to +1.3 \\
\hline Woods Cove (WDS) & $\begin{array}{l}33^{\circ} 31^{\prime} 42^{\prime \prime} \mathrm{N} \\
117^{\circ} 46^{\prime} 05^{\prime \prime} \mathrm{W}\end{array}$ & Tso & $\begin{array}{l}\text { Flattened benches separated by } \\
\text { channels }\end{array}$ & +1.0 to +1.5 \\
\hline Victoria Beach (VIC) & $\begin{array}{l}33^{\circ} 31^{\prime} 11^{\prime \prime} \mathrm{N} \\
117^{\circ} 45^{\prime} 52^{\prime \prime} \mathrm{W}\end{array}$ & Tso & $\begin{array}{l}\text { Flattened benches separated by } \\
\text { channels }\end{array}$ & +1.2 to +1.5 \\
\hline Treasure Island (TRI) & $\begin{array}{l}33^{\circ} 30^{\prime} 48^{\prime \prime} \mathrm{N} \\
117^{\circ} 45^{\prime} 33^{\prime \prime} \mathrm{W}\end{array}$ & Tso & $\begin{array}{l}\text { Gently sloping benches separated by } \\
\text { tidal pools and channels }\end{array}$ & +1.1 to +1.3 \\
\hline Thousand Steps (THS) & $\begin{array}{l}33^{\circ} 29^{\prime} 55^{\prime \prime} \mathrm{N} \\
117^{\circ} 44^{\prime} 35^{\prime \prime} \mathrm{W}\end{array}$ & Tso & $\begin{array}{l}\text { Granitic boulders, including larger, } \\
\text { horizontal rocks supporting } \\
\text { rockweeds, separated by channels }\end{array}$ & +0.8 to +1.1 \\
\hline$\dagger$ Monarch Bay (MBY) & $\begin{array}{l}33^{\circ} 29^{\prime} 03^{\prime \prime} \mathrm{N} \\
117^{\circ} 43^{\prime} 56^{\prime \prime} \mathrm{W}\end{array}$ & $\mathrm{T}$ & $\begin{array}{l}\text { Flattened, benches with occasional } \\
\text { channels and tidal pools }\end{array}$ & +0.7 to +1.2 \\
\hline$\dagger$ Dana Point (DPT) & $\begin{array}{l}33^{\circ} 27^{\prime} 35^{\prime \prime} \mathrm{N} \\
117^{\circ} 42^{\prime} 52^{\prime \prime} \mathrm{W}\end{array}$ & Tso & $\begin{array}{l}\text { Granitic boulders mixed with } \\
\text { flattened benches }\end{array}$ & +0.9 to +1.3 \\
\hline
\end{tabular}

" Sites located within intertidal MPAs designated between 1969 and 1972 are indicated by a $\uparrow$. Remaining sites were all accorded similar MPA status in 1994 but sites were not signed and the public was largely unaware of this change in status during the study.

${ }^{b}$ See Figure 1 for site locations.

c Tm, Monterey Formation in the Tertiary Period; Tso, San Onofre Breccia in the Tertiary Period.

invertebrates has been prohibited for more than $25 \mathrm{yr}$ (McArdle 1997). The remaining four sites were accorded similar MPA status on 1 January 1994. Previous research (Murray 1998, Murray et al. 1999) has shown that the extraction of intertidal invertebrates is common throughout the region and occurs even in these long-standing MPAs.

\section{Environmental Parameters}

Selected environmental parameters were investigated to determine possible sources of among-site variation in the structure of subcanopy communities. The tidal heights of all sampled areas were determined using standard surveying techniques and reference points established at each site using predicted tidal data. Variation in wave exposure was estimated by quantifying maximum wave force using a modification of procedures described by Bell and Denny (1994). Three wave force meters, separated by at least $2.0 \mathrm{~m}$, were deployed at each site on days when local wave conditions were judged to be representative of the study area. Assessments were performed simultaneously at multiple sites (3-6), including a reference site at Crystal Cove, between 19 and 25 December 1996, and 30 April and 11 June 1997. Meters were placed at each site on at least three different days during each assessment period and were deployed during flood tide and retrieved the following morning. Upon retrieval, the maximum wave force was obtained from calibration tables developed for each meter. Sand movement interferes with hydrodynamic drag on the transducer housing and can affect acceleration of the drogue ball (Bell and Denny 1994). Hence, after deployment, estimates of sand accumulation were made for each meter using an arbitrary ranking system ranging from 0 to 10:0 indicated the absence of sand, and 10 indicated that sand completely filled the transducer housing. Wave force measurements were accepted only when sand values were less than five. Data were analyzed using both the measured wave force velocity 
TABLE 2

Patterns of Human Visitation during Low Tides $(\leq+1.0 \mathrm{ft}[0.3 \mathrm{~m}])$ at the Study Sites: Listed Are Shoreline Spans for the Study Areas Within Each Site and Numbers of Low-Tide Visitors

\begin{tabular}{lcccc}
\hline \hline & $\begin{array}{c}\text { Shoreline } \\
\text { Span }\end{array}$ & $\begin{array}{c}\text { Visitors per } \\
\text { 10-min } \\
\text { Observation }\end{array}$ \\
Site $^{n}$ (Abbreviation) & (Linear m) & Annual Mean) & $\begin{array}{c}\text { Visitors per } \\
\text { 10 m of } \\
\text { Shoreline }\end{array}$ & $\begin{array}{c}\text { Ranks for } \\
\text { Visitor } \\
\text { Disturbance }\end{array}$ \\
\hline †Crystal Cove (CRC) & 87.8 & 2.6 & 0.30 & 5 \\
†haw's Cove (SHW) & 209.4 & 7.4 & 0.35 & 4 \\
Woods Cove (WDS) & 78.2 & 7.4 & 0.95 & 1 \\
Victoria Beach (VTC) & 100.4 & 4.4 & 0.44 & 3 \\
Treasure Island (TRI) & 61.7 & 3.0 & 0.49 & 2 \\
Thousand Steps (THS) & 53.9 & 1.6 & 0.30 & 5 \\
†Monarch Bay (MBY) & 73.5 & 0.4 & 0.05 & 8 \\
$\dagger$ Dana Point (DPT) & 321.0 & 8.8 & 0.27 & 7 \\
\hline
\end{tabular}

a Sites located within intertidal MPAs designated between 1969 and 1972 are indicated by a $\dagger$.

${ }^{b}$ Numbers of visitors are based on five 10-min observations made four days per month from February 1995 through January 1996 (see text for explanation).

' Ranks for visitor disturbance are based on numbers per 10 -min period per $10 \mathrm{~m}$ of shoreline.

$(\mathrm{m} \mathrm{sec})^{-1}$ ) and velocities standardized using values recorded during the same tidal cycle at the Crystal Cove reference site. Because both methods yielded highly similar results, only actual measured velocities are reported here.

Sand scour and deposition are important components shaping intertidal community structure in southern California (Murray and Bray 1993). Because sand movements are transitory and difficult to quantify (Stewart 1983, 'Trowbridge 1996), three methods were used to determine the degree of sand influence at each site: (1) sand cover was estimated for sampled quadrats; (2) sand accumulation was recorded in wave force housings; and (3) sites were ranked based on qualitative observations of the amount of sand present throughout the study period. Sand cover was estimated for 10 randomly distributed quadrats in the Silvetia compressa assemblage at each site during both autumn 1995 and spring 1996. Cover estimates were made directly in the field using a random point contact method described later in this section, or in the laboratory by inspecting video records of quadrats taken with a $\mathrm{Hi}-8 \mathrm{~mm}$ camcorder (Sony Hi-8 CCD-VX3). Sand accumulation within wave force meters was ranked as described previously and the median value for each site determined. Last, sites were ranked using subjective notes detailing sand presence over all seasons and tidal cycles over the 3-yr study period.

The study sites experience different and generally high levels of human use throughout the year (Murray 1998, Murray et al. 1999; Table 2). Data depicting the levels of human visitation were available for each site based on studies performed from February 1995 to January 1996 (Murray et al. 1999; S.N.M., unpubl. data). In these studies, the number of visitors at low tide was determined over a prescribed linear section of shoreline four times per month for 12 consecutive months. All observations were made between dawn and dusk during lower low tides $(\leq 1.0$ $\mathrm{ft}[0.3 \mathrm{~m}]$ with reference to MLLW [Mean Lower Low Water]). During each observation period, the number of shoreline visitors was determined for five 10 -min periods completed at approximately $30-\mathrm{min}$ intervals beginning $1 \mathrm{hr}$ before and ending $1 \mathrm{hr}$ after the predicted time of low water. Site observations were evenly divided each month between weekend and midweek days. Based on these data, the number of shore visitors per $10 \mathrm{~m}$ of shoreline was calculated and used to represent the exposure of each site to human disturbance (Table 2). These studies revealed that Woods Cove, Victoria Beach, and Treasure 
Island receive the greatest concentrations of shore visitors. Intermediate levels of human use occur at Crystal Cove, Shaw's Cove, and Thousand Steps, and Monarch Bay and Dana Point receive the lowest numbers of visitors.

\section{Biological Sampling Methods}

Spatial variation in the structure of the Silvetia compressa subcanopy assemblage was assessed during the autumn and spring. At each site, twenty 0.3 by $0.5 \mathrm{~m}\left(0.15 \mathrm{~m}^{2}\right)$ quadrats were randomly located on largely horizontal surfaces supporting at least $80 \%$ S. compressa canopy cover. The center of each quadrat was marked with a stainless-steel bolt, which was anchored into the substratum with drop anchors or marine epoxy. Ten quadrats were sampled during the autumn (23 September to 4 December 1995) and 10 during the spring (8 March to 7 June 1996), 5.5 to 6.5 months after autumn assessments.

Overstory rockweed cover was determined by visual scanning after subdividing each quadrat into four equal sections to facilitate estimates. The cover of seaweeds and sessile invertebrates in the understory assemblage was then sampled using a random point contact (RPC) method modeled after procedures described by Foster et al. (1991a). Points (100) were randomly located within quadrats using a leveled Plexiglas (=Perspex) platform (35 by 50 by $1.2 \mathrm{~cm}$ ) containing 150 holes (3.1 $\mathrm{mm}$ diameter) spaced at 3.3 -cm intervals and distributed in 10 rows and 15 columns. Platforms were leveled using three adjustable legs, positioned 0.2 to $0.5 \mathrm{~m}$ above the substratum, and a $3.0-\mathrm{mmd}$ stainless-steel rod was dropped through each hole. If multiple species were contacted by the path of a rod, then each was recorded once for that point. Percentage cover was calculated as the number of contacts divided by the total number of rods dropped on the quadrat. Therefore, it was possible for total quadrat cover to exceed $100 \%$, as often occurs in complex, threedimensional intertidal communities. Mobile invertebrate species were counted to obtain densities. Each quadrat also was carefully searched to obtain a full species inventory. Species present, but not contacted by rods, were arbitrarily assigned a cover value of $0.5 \%$ to facilitate data analysis. Where morphologically similar species could not be consistently distinguished with accuracy (e.g., Corallina pinnatifolia and $C$. vancouveriensis, Macclintockia scabra and Lottia conus), they were combined and treated in our analyses as a single taxon. Species also were assigned to suprataxa (crustose algae, articulated coralline algae, frondose [branched and bladed] algae, sessile macroinvertebrates, and limpets + chitons) for further examination of distributional and abundance patterns. Attempts were made to correlate the abundances of suprataxa with each other and with variations in quadrat-scale environmental variables.

Species presence and abundance data formed the basis for the site and temporal comparisons. Site diversity was calculated separately for macrophytes and sessile macroinvertebrates based on cover data and for mobile macroinvertebrates using density data. Diversity was expressed as richness $(S)$, defined here as the number of discriminated taxa, and as Shannon's $H^{\prime}$ Index (Shannon and Weaver 1949). Cluster analysis and nonmetric multidimensional scaling (MDS) were used to examine spatial and temporal patterns in the subcanopy assemblage. Relationships between environmental data and site ordination patterns were probed by qualitatively comparing patterns in measured abiotic parameters with site ordination results.

\section{Statistical Methods}

A one-way analysis of variance (ANOVA) model was used to test for differences in species abundances among sites. Patterns of spatial and temporal variation in wave force were examined using a two-way ANOVA design. Data sets were first examined for homoscedasticity using Cochran's test, transformed if necessary to meet variance requirements, and subjected to ANOVA when criteria for parametric analysis could be satisfied. ANOVA is robust to nonnormality (Underwood 1997); therefore, departures from normality were not considered reason to reject parametric procedures. When the homoscedasticity criterion could not be met by 
transformation and variance departures were judged to be large, a randomization test was used to test the null hypothesis of no difference among groups in lieu of a one-way ANOVA model. As suggested by Manly (1997), random reassignment of the data was repeated 1000 times for setting $\alpha$ at 0.05 .

Regional differences in species abundances between the autumn and spring sampling periods were further analyzed using a paired $t$-test with the mean values for each site serving as the paired replicates; however, if variance equality could not be satisfied, Welch's approximate $t$ was employed. This is a more conservative and reliable test than the traditional $t$-test under these circumstances (Quinn and Keough 2002). The nonparametric Spearman's correlation procedure was used to identify relationships between suprataxa and between suprataxa and abiotic variables (quadrat sand cover and tidal height) using the full set of quadrat samples $(n=160)$ and between diversity values and environmental parameters using mean site values $(n=8)$.

Among-site and sampling period variations in the structure of the Silvetia compressa understory assemblage were analyzed by cluster analysis and MDS ordination. Mean cover of subcanopy macrophytes and macroinvertebrates was calculated for each site for both autumn 1995 and spring 1996. Dendrograms depicting autumn and spring site and species clusters were constructed from association matrices based on the Bray-Curtis similarity index (Bray and Curtis 1957) using the unweighted pair group average clustering algorithm. The similarity percentages (SIMPER) procedure (Clarke and Warwick 2001) was used to examine the contributions of individual species to within-group similarity and between-group dissimilarity. Ordinations by MDS of the eight sites were performed using both cover (seaweeds and sessile invertebrates) and density (mobile invertebrates) data. Untransformed cover data were used for both cluster and MDS analyses, and density data were standardized to produce relative abundances before performing MDS. Because the data sets contained a very large number of rare (low-abundance) spe- cies, only the 25 most abundant taxa were used for the cluster and MDS analyses.

ANOSIM tests (Clarke and Green 1988) were used to assess differences between longstanding and recently established MPÁs (one-way ANOSIM) and among sites and between the seasonal sampling periods (twoway crossed ANOSIM) using community cover and density data for all taxa. Separate analyses were performed to test for seasonal differences in the biotic assemblages of the two MPA site groups because of the very large number of permutations contained in the data sets. Symbols scaled in size according to the values of tested environmental variables were superimposed on the cover and density MDS ordinations to qualitatively examine relationships between site patterns and environmental parameters using routines provided by PRIMER.

All univariate statistical analyses were performed using BIOSTAT I computer programs (Pimentel and Smith 1990). Multivariate statistical procedures and diversity calculations were executed using PRIMER (Clarke and Gorley 2001, Clarke and Warwick 2001).

\section{RESULTS}

\section{Abiotic Variables}

Maximum wave force differed significantly among sites, with an interaction between site and season (Table 3). The greatest mean forces were recorded at Woods Cove, Victoria Beach, and Treasure Island, the three sites located in the central part of the study region (Figure 1). Lowest forces occurred at Crystal Cove and Shaw's Cove, the two most northwestern sites. Greater wave force was measured during spring at all sites except Victoria Beach and Treasure Island, where maximum wave force was greatest during autumn. Thousand Steps was exposed to the greatest overall sand influence; high sand influence also occurred at Monarch Bay and Shaw's Cove (Table 4). Least sand influence occurred at Treasure Island, Woods Cove, and Victoria Beach, the same three sites exposed to the greatest wave energy. 
TABLE 3

Maximum Wave Force at the Study Sites as Determined by Described Methodologies

\begin{tabular}{|c|c|c|c|c|c|}
\hline \multirow[b]{3}{*}{ Site $^{a}$} & \multicolumn{5}{|c|}{ Maximum Wave Force $b$} \\
\hline & \multicolumn{2}{|c|}{ Autumn 1996} & \multicolumn{2}{|c|}{ Spring 1997} & \multirow{2}{*}{$\frac{\text { Average }}{\left(\mathrm{m} \mathrm{sec}^{-1}\right)}$} \\
\hline & $n$ & $\left(m \sec ^{-1}\right)$ & $n$ & $\left.(\mathrm{~m} \mathrm{sec})^{-1}\right)$ & \\
\hline fCrystal Cove & 17 & $4.63 \pm 0.08$ & 19 & $5.12 \pm 0.10$ & $4.89 \pm 0.08$ \\
\hline †Shaw's Cove & 6 & $4.66 \pm 0.27$ & 8 & $5.00 \pm 0.21$ & $4.86 \pm 0.17$ \\
\hline Woods Cove & 6 & $6.60 \pm 0.46$ & 6 & $7.84 \pm 1.02$ & $7.22 \pm 0.57$ \\
\hline Victoria Beach & 10 & $7.55 \pm 0.40$ & 11 & $6.20 \pm 0.47$ & $6.84 \pm 0.34$ \\
\hline Treasure Island & 9 & $6.45 \pm 0.22$ & 7 & $5.95 \pm 0.51$ & $6.23 \pm 0.25$ \\
\hline Thousand Steps & 5 & $4.65 \pm 0.15$ & 5 & $5.21 \pm 0.17$ & $4.93 \pm 0.14$ \\
\hline †Monarch Bay & 4 & $4.79 \pm 0.39$ & 9 & $5.33 \pm 0.25$ & $5.17 \pm 0.21$ \\
\hline †Dana Point & 9 & $4.48 \pm 0.15$ & 9 & $5.82 \pm 0.42$ & $5.15 \pm 0.27$ \\
\hline ANOVA results & $\mathrm{df}$ & $F$ & & & \\
\hline Site & 7,124 & 15.132 & & & \\
\hline Season & 1,124 & 3.268 & & & \\
\hline Site $\times$ Season & 7,124 & 4.125 & & & \\
\hline
\end{tabular}

"Sites located within intertidal MPAs designated between 1969 and 1972 are indicated by a $\neq$.

${ }_{b}$ Two-way ANOVA models were used to analyze wave force data where both site and season were treated as fixed factors. Values reported are the means $( \pm 1 \mathrm{SE})$ for the indicated number of replicates, and the degrees of freedom (df) and $F$ values for the ANOVA tests. Significant differences are indicated by asterisks: ${ }^{* *}, P<0.001$.

\section{TABLE 4}

Sand Influence at the Study Sites

\begin{tabular}{|c|c|c|c|c|c|c|c|c|c|c|}
\hline \multirow[b]{2}{*}{ Site $^{a}$} & \multicolumn{4}{|c|}{ Quadrat Sand Cover } & \multicolumn{4}{|c|}{ Sand Accumulation in Wave Meters } & \multirow{2}{*}{$\begin{array}{l}\text { Qualitative } \\
\text { Evaluation } \\
\text { of Sand } \\
\text { Influence } \\
\text { Rank }\end{array}$} & \multirow{2}{*}{$\begin{array}{c}\text { Overall } \\
\text { Sand } \\
\text { Influence } \\
\text { Site } \\
\text { Rank }\end{array}$} \\
\hline & $\begin{array}{c}\text { Autumn } \\
1995(\%)\end{array}$ & $\begin{array}{c}\text { Spring } \\
1996(\%)\end{array}$ & $\begin{array}{l}\text { Combined } \\
\text { Autumn + } \\
\text { Spring (\%) }\end{array}$ & $\begin{array}{l}\text { Site } \\
\text { Rank }\end{array}$ & $\begin{array}{c}\text { Autumn } \\
1996 \\
\text { Rank }\end{array}$ & $\begin{array}{c}\text { Spring } \\
1997 \\
\text { Rank }\end{array}$ & $\begin{array}{c}\text { Mean } \\
\text { 1996-1997 } \\
\text { Rank }\end{array}$ & $\begin{array}{l}\text { Site } \\
\text { Rank }\end{array}$ & & \\
\hline $\begin{array}{c}\text { †Crystal } \\
\text { Cove }\end{array}$ & $10.0 \pm 4.8$ & $22.5 \pm 4.7$ & $16.3 \pm 3.6$ & 4 & 4 & 5.5 & 4.75 & 5 & 5 & 4.5 \\
\hline $\begin{array}{c}\text { †Shaw's } \\
\text { Cove }\end{array}$ & $7.4 \pm 3.0$ & $14.4 \pm 4.5$ & $10.9 \pm 2.8$ & 6 & 4 & 3 & 3.5 & 3 & 3 & 3 \\
\hline $\begin{array}{c}\text { Woods } \\
\text { Cove }\end{array}$ & $0.2 \pm 0.2$ & $0.8 \pm 0.5$ & $0.5 \pm 0.3$ & 8 & 4 & 5.5 & 4.75 & 5 & 6 & 7 \\
\hline $\begin{array}{r}\text { Victoria } \\
\text { Beach }\end{array}$ & $21.9 \pm 7.6$ & $6.8 \pm 2.7$ & $14.4 \pm 4.3$ & 5 & 4 & 5.5 & 4.75 & 5 & 7 & 6 \\
\hline $\begin{array}{c}\text { Treasure } \\
\text { Island }\end{array}$ & $3.2 \pm 1.5$ & 0.0 & $1.6 \pm 0.8$ & 7 & 7.5 & 8 & 7.75 & 8 & 8 & 8 \\
\hline $\begin{array}{l}\text { Thousand } \\
\text { Steps }\end{array}$ & $31.1 \pm 5.2$ & $9.7 \pm 4.8$ & $20.4 \pm 4.2$ & 2 & 1 & 2 & 1.5 & 1 & 1 & 1 \\
\hline $\begin{array}{l}\$ \text { Monarch } \\
\text { Bay }\end{array}$ & $19.1 \pm 4.0$ & $24.5 \pm 5.0$ & $21.8 \pm 3.2$ & 1 & 4 & 1 & 2.5 & 2 & 2 & 2 \\
\hline $\begin{array}{l}\text { TDana } \\
\text { Point }\end{array}$ & $16.4 \pm 4.0$ & $16.6 \pm 5.7$ & $16.5 \pm 3.3$ & 3 & 7.5 & 5.5 & 6.5 & 7 & 4 & 4.5 \\
\hline
\end{tabular}

Note: Values reported are mean $( \pm 1 \mathrm{SE})$ sand cover estimated from quadrats, rankings based on median values for sand accumulation in wave meter housings, and qualitative evaluations of sand cover at the sites. See text for explanations. Overall site rankings are based on mean rankings of the three procedures.

a Sites located within intertidal MPAs designated between 1969 and 1972 are indicated by a $\uparrow$. 
TABLE 5

Mean Rockweed Cover for the Study Sites

\begin{tabular}{|c|c|c|c|c|c|c|c|c|c|}
\hline \multirow[b]{2}{*}{ Seaweed Taxa } & \multicolumn{8}{|c|}{ Sites ${ }^{a}$} & \multirow{2}{*}{$\begin{array}{c}\text { Overall } \\
\text { Mean }\end{array}$} \\
\hline & tCRC & $\dashv \mathrm{SHW}$ & WDS & VIC & TRI & THS & $\dagger \mathrm{MBY}$ & tDPT & \\
\hline \multicolumn{10}{|l|}{ Autumn 1995} \\
\hline \multicolumn{10}{|l|}{$\begin{array}{l}\text { Silvetia compressa (J. Ag.) Serrão, } \\
\text { Cho \& Brawley }\end{array}$} \\
\hline Overstory canopy & $\begin{array}{c}94.3 \\
( \pm 2.6)\end{array}$ & $\begin{array}{c}97.2 \\
( \pm 0.7)\end{array}$ & $\begin{array}{c}80.2 \\
( \pm 2.2)\end{array}$ & $\begin{array}{r}92.6 \\
( \pm 2.7)\end{array}$ & $\begin{array}{c}87.5 \\
( \pm 4.3)\end{array}$ & $\begin{array}{c}93.3 \\
( \pm 1.4)\end{array}$ & $\begin{array}{c}98.9 \\
( \pm 0.6)\end{array}$ & $\begin{array}{c}96.3 \\
( \pm 1.0)\end{array}$ & $192.5^{\star * * *}$ \\
\hline Understory holdfast & $\begin{array}{c}4.0 \\
( \pm 0.6)\end{array}$ & $\begin{array}{r}4.6 \\
( \pm 0.5)\end{array}$ & $\begin{array}{c}4.7 \\
( \pm 0.7)\end{array}$ & $\begin{array}{r}7.6 \\
( \pm 1.2)\end{array}$ & $\begin{array}{c}9.1 \\
( \pm 1.8)\end{array}$ & $\begin{array}{r}5.8 \\
( \pm 0.9)\end{array}$ & $\begin{array}{r}3.2 \\
( \pm 0.4)\end{array}$ & $\begin{array}{c}5.5 \\
( \pm 0.9)\end{array}$ & ${ }^{1} 5.6^{\star \star}$ \\
\hline $\begin{array}{l}\text { Total S. compressa cover } \\
\text { Hesperopbycus califormicus Silva }\end{array}$ & 98.3 & 101.8 & 84.9 & 100.2 & 96.6 & 99.1 & 102.1 & 101.8 & 98.1 \\
\hline Overstory canopy & & 1.3 & & & 0.8 & & & & 0.3 \\
\hline Understory holdfast & & & & & 0.3 & & 0.2 & & $<0.1$ \\
\hline Total cover & & 1.3 & & & 1.1 & & 0.2 & & $<0.1$ \\
\hline \multicolumn{10}{|l|}{ Spring 1996} \\
\hline \multicolumn{10}{|l|}{$\begin{array}{l}\text { Silvetia compressa (J. Ag.) Serrão, } \\
\text { Cho \& Brawley }\end{array}$} \\
\hline Overstory canopy & $\begin{array}{r}91.5 \\
( \pm 2.4)\end{array}$ & $\begin{array}{r}84.5 \\
( \pm 3.1)\end{array}$ & $\begin{array}{c}80.5 \\
( \pm 3.1)\end{array}$ & $\begin{array}{c}90.2 \\
( \pm 1.9)\end{array}$ & $\begin{array}{c}85.2 \\
( \pm 3.7)\end{array}$ & $\begin{array}{r}74.4 \\
( \pm 2.8)\end{array}$ & $\begin{array}{c}96.5 \\
( \pm 1.0)\end{array}$ & $\begin{array}{c}90.8 \\
( \pm 1.9)\end{array}$ & $86.7^{* * *}$ \\
\hline Understory holdfast & $\begin{array}{c}5.7 \\
( \pm 0.9)\end{array}$ & $\begin{array}{r}3.9 \\
( \pm 0.5)\end{array}$ & $\begin{array}{r}4.8 \\
( \pm 0.5)\end{array}$ & $\begin{array}{c}6.7 \\
( \pm 0.8)\end{array}$ & $\begin{array}{r}6.6 \\
( \pm 1.0)\end{array}$ & $\begin{array}{c}3.3 \\
( \pm 0.4)\end{array}$ & $\begin{array}{r}3.6 \\
( \pm 0.7)\end{array}$ & $\begin{array}{r}6.6 \\
( \pm 1.0)\end{array}$ & $5.2^{* *}$ \\
\hline $\begin{array}{c}\text { Total S. compressa cover } \\
\text { Hesperopbycus californicus Silva }\end{array}$ & 97.2 & 88.4 & 85.3 & 96.9 & 91.8 & 77.7 & 100.1 & 97.5 & 91.8 \\
\hline Overstory canopy & & & & 0.2 & & & & & $<0.1$ \\
\hline Understory holdfast & & & & 0.1 & & & & & $<0.1$ \\
\hline Total cover & & & & 0.3 & & & & & \\
\hline
\end{tabular}

Note: Among-site differences assessed by one-way ANOVA. Values reported are site means ( \pm 1 SE) for 10 replicate plots. Superscript $\left({ }^{1}\right)$ indicates data were transformed before analyses. Significant differences among sites are indicated by asterisks: ${ }_{\star \star \star}, P<0.01$; ***, $P<0.001$

a Sites located within intertidal MPAs designated between 1969 and 1972 are indicated by a $\uparrow$.

\section{Rockweed Canopy Cover}

Silvetia compressa canopy cover varied significantly among sites during both sampling periods (Table 5). Mean canopy cover was significantly greater in the autumn $(t=2.526$, $P=0.04$ ) but then decreased by spring at seven of the eight sites (Table 5). Greatest canopy cover occurred at Monarch Bay, a site with high sand influence and lowest levels of human disturbance, and the least cover at Woods Cove and Thousand Steps. Hesperophycus californicus, another upper-shore rockweed, was rare throughout the study area and occurred in quadrats at only four sites, where it never provided more than $1.3 \%$ cover (Table 5).

Morphologies of Silvetia compressa thalli were qualitatively similar except at Monarch
Bay where frond lengths two to three times greater than those found at our other study sites were common. Holdfast area ranged from 3.2 to $9.1 \%$ and varied significantly among sites; however, mean holdfast cover did not differ significantly between assessment periods ( $t=0.755, P=0.52$ ). Monarch Bay thalli were the least densely aggregated and exhibited a canopy-to-holdfast cover ratio of nearly 30 to 1 . The lowest canopy-toholdfast cover ratios $(<13: 1)$ and the densest aggregations of $S$. compressa thalli were found at Victoria Beach and Treasure Island.

\section{Structure of the Understory Assemblage}

Seaweeds contributed $71.2 \%$ of the subcanopy cover averaged over all sites compared with $23.8 \%$ sessile invertebrate cover. Mobile 
TABLE 6

Mean Cover for Abundant Crustose, Articulated Coralline, and Frondose Algae for Autumn 1995

\begin{tabular}{|c|c|c|c|c|c|c|c|c|c|}
\hline \multirow[b]{2}{*}{ Seaweed Taxa } & \multicolumn{8}{|c|}{ Sites $^{a}$} & \multirow[b]{2}{*}{ Mean $\pm \mathrm{SE}$} \\
\hline & $\dagger \mathrm{CRC}$ & $+\mathrm{SHW}$ & WDS & VIC & TRI & THS & $\uparrow \mathrm{MBY}$ & $\dagger \mathrm{DPT}$ & \\
\hline \multicolumn{10}{|l|}{ Crustose algae } \\
\hline $\begin{array}{l}\text { Pseudolitboderna nigra } \\
\text { Hollenb. }\end{array}$ & 20.2 & 45.3 & 11.3 & 25.2 & 34.8 & 50.8 & 21.7 & 38.5 & $31.0 \pm 4.8^{\star \star \star}$ \\
\hline $\begin{array}{l}\text { Pseudolitbophyllum } \\
\text { neofarlowii (Setch. \& } \\
\text { Mason) Adey }\end{array}$ & 15.1 & 18.4 & 24.8 & 23.0 & 21.7 & 17.2 & 18.0 & 28.8 & $20.9 \pm 1.6 \mathrm{~ns}$ \\
\hline $\begin{array}{l}\text { Unidentified crustose } \\
\text { coralline }\end{array}$ & 1.4 & 0.1 & 1.0 & & 1.0 & 0.1 & 5.5 & & ${ }^{2} 1.1 \pm 0.8^{* *}$ \\
\hline $\begin{array}{l}\text { Unidentified } \\
\text { Peyssonneliaceae/ } \\
\text { Hildenbrandiaceae }\end{array}$ & 0.2 & 1.7 & 1.2 & 0.3 & 0.8 & 0.9 & 0.6 & 0.3 & $0.8 \pm 0.2 \mathrm{~ns}$ \\
\hline Other crustose species $(n=3)$ & 1.5 & 0.1 & 0.9 & 0.5 & 1.1 & 0.1 & & 0.1 & $0.6 \pm 0.5$ \\
\hline Total crustose algae cover & 38.4 & 65.6 & 39.2 & 49.0 & 59.4 & 69.1 & 45.8 & 67.7 & $54.3 \pm 4.5$ \\
\hline Articulated coralline algae & & & & & & & & & \\
\hline $\begin{array}{l}\text { Corallina pinnatifolia (Manza) } \\
\text { Daws. and } C \text {. vancouveriensis } \\
\text { Yendo }\end{array}$ & 10.1 & 4.0 & 12.1 & 1.5 & 7.9 & 6.7 & 36.7 & 11.6 & ${ }^{1} 11.2 \pm 3.9^{\star * *}$ \\
\hline Fania crassa Lamour. & 1.4 & & & & & & 2.9 & 0.6 & ${ }^{2} 0.6 \pm 0.7^{\star \star \star}$ \\
\hline $\begin{array}{l}\text { Other articulated coralline } \\
\text { species }(n=3)\end{array}$ & 0.1 & & 0.1 & & 0.1 & 0.1 & 0.2 & & $0.2 \pm 0.2$ \\
\hline $\begin{array}{l}\text { Total articulated coralline } \\
\text { algae cover }\end{array}$ & 11.6 & 4.0 & 12.2 & 1.5 & 8.0 & 6.8 & 39.8 & 12.2 & $12.0 \pm 4.2$ \\
\hline \multicolumn{10}{|l|}{ Frondose algae } \\
\hline $\begin{array}{l}\text { Gelidium pusillum (Stackh.) Le } \\
\text { Jol. and G. coulteri Harv. }\end{array}$ & 2.1 & 0.1 & 0.9 & 0.1 & & 1.1 & 2.5 & 4.4 & ${ }^{2} 1.1 \pm 0.5^{\star}$ \\
\hline Cryptopleura crispa Kyl. & 0.2 & 0.1 & 0.1 & & 0.4 & 0.1 & 1.4 & 1.0 & ${ }^{2} 0.4 \pm 0.2 \mathrm{~ns}$ \\
\hline $\begin{array}{l}\text { Chondracantbus canaliculatus } \\
\text { (Harv.) Guiry }\end{array}$ & 0.4 & 0.1 & 0.5 & & & 0.2 & 1.1 & 0.6 & ${ }^{2} 0.3 \pm 0.2 \mathrm{~ns}$ \\
\hline $\begin{array}{l}\text { Other frondose algae species } \\
\quad(n=26)\end{array}$ & 5.8 & 0.1 & 0.4 & 0.1 & 0.4 & 1.1 & 2.7 & 2.8 & $1.8 \pm 1.8$ \\
\hline Total frondose algae cover & 8.4 & 0.4 & 1.9 & 0.2 & 0.9 & 2.5 & 7.7 & 8.9 & $4.0 \pm 3.5$ \\
\hline Total understory seaweed cover & 58.4 & 70.0 & 53.3 & 50.7 & 68.3 & 78.4 & 93.3 & 88.8 & $70.1 \pm 5.6$ \\
\hline
\end{tabular}

Note: Superscripts indicate data were transformed before ANOVA analyses (') or were subjected to a randomization test following evaluation of variance ratios $\left({ }^{2}\right)$. Only the Gelidium pusillum and G. coulteri data showed unequal population dispersions based on the ratio of the largest to the smallest variance so this randomization test result should be interpreted with caution. Significant differences among sites are indicated by asterisks: ${ }^{*}, P<0.05 ;{ }^{* *}, P<0.01 ;{ }^{* * *}, P<0.001$.

"Sites located within intertidal MPAs designated between 1969 and 1972 are indicated by a $f$.

invertebrates contributed an average understory density of $363.9 \mathrm{~m}^{-2}$ over all sites.

SEAWEEDS. Mean seaweed cover in the subcanopy assemblage ranged from 50.7 to $94.4 \%$ and varied significantly among sites but did not differ between sampling periods (Tables 6,7). Greatest cover was found at Monarch Bay, the site characterized by lesscrowded aggregations of larger Silvetia com- pressa thalli. Least cover occurred at Victoria Beach, a site that supported denser S. compressa aggregations and received higher wave force.

Of the 45 subcanopy seaweeds recorded during the study, three (Pseudolitboderma nigra, Pseudolitbophyllum neofarlowii, and Corallina pinnatifolia/C. vancouveriensis) accounted for nearly two-thirds of total understory 
TABLE 7

Mean Cover for Abundant Crustose, Articulated Coralline, and Frondose Algae for Spring 1996

\begin{tabular}{|c|c|c|c|c|c|c|c|c|c|}
\hline \multirow[b]{2}{*}{ Seaweed Taxa } & \multicolumn{8}{|c|}{ Sites $^{a}$} & \multirow[b]{2}{*}{ Mean $\pm \mathrm{SE}$} \\
\hline & †CRC & $\uparrow \mathrm{SHW}$ & WDS & VIC & TRI & THS & $\uparrow \mathrm{MBY}$ & $\dagger \mathrm{DPT}$ & \\
\hline \multicolumn{10}{|l|}{ Crustose algae } \\
\hline Pseudolitboderma nigra Hollenb. & 29.8 & 32.0 & 14.3 & 35.3 & 41.6 & 43.4 & 15.5 & 36.6 & $31.0 \pm 3.9^{* *}$ \\
\hline $\begin{array}{l}\text { Pseudolithopbyllum neofarlowii } \\
\text { (Setch. \& Mason) Adey }\end{array}$ & 21.0 & 22.2 & 19.1 & 19.3 & 21.8 & 10.2 & 25.1 & 20.4 & $19.9 \pm 1.5 \mathrm{~ns}$ \\
\hline Unidentified crustose coralline & 1.3 & 5.1 & 4.2 & & 1.2 & 0.1 & 2.6 & 1.6 & $22.0 \pm 0.7^{* * *}$ \\
\hline $\begin{array}{l}\text { Unidentified Peyssonneliaceae/ } \\
\text { Hildenbrandiaceae }\end{array}$ & 0.4 & 3.5 & 3.6 & 1.5 & 0.6 & 2.7 & 1.0 & 1.8 & ${ }^{\mathrm{I}} 1.9 \pm 0.4^{*}$ \\
\hline Other crustose algal species $(n=3)$ & 0.2 & 0.3 & 1.7 & & & 0.3 & 0.1 & 0.7 & $0.4 \pm 0.5$ \\
\hline Total crustose algae cover & 52.7 & 63.1 & 42.9 & 56.1 & 65.2 & 56.7 & 44.3 & 61.1 & $55.2 \pm 2.9$ \\
\hline \multicolumn{10}{|l|}{ Articulated coralline algae } \\
\hline $\begin{array}{l}\text { Corallina pinnatifolia (Manza) Daws. } \\
\text { and C. vancouveriensis Yendo }\end{array}$ & 8.3 & 14.4 & 11.7 & 0.8 & 7.2 & 4.1 & 34.4 & 8.2 & ${ }^{1} 10.9 \pm 3.7^{* * *}$ \\
\hline 7ania crassa Lamour. & 1.2 & & & & & & 6.1 & 0.8 & ${ }^{2} 1.0 \pm 1.7^{\star * *}$ \\
\hline $\begin{array}{l}\text { Other articulated coralline algal } \\
\text { species }(n=2)\end{array}$ & & 0.1 & & & & & 0.1 & & $0.2 \pm 0.2$ \\
\hline $\begin{array}{l}\text { Total articulated coralline algae } \\
\text { cover }\end{array}$ & 9.5 & 14.5 & 11.7 & 0.8 & 7.2 & 4.1 & 40.6 & 9.0 & $12.1 \pm 4.3$ \\
\hline \multicolumn{10}{|l|}{ Frondose algae } \\
\hline $\begin{array}{l}\text { Gelidium pusillum (Stackh.) Le Jol. } \\
\text { and G. coulteri Harv. }\end{array}$ & 2.8 & 1.6 & 0.7 & 0.2 & 0.2 & 0.1 & 2.5 & 3.2 & ${ }^{1} 1.1 \pm 0.4^{\star *}$ \\
\hline $\begin{array}{l}\text { Cbondracantbus canaliculatus (Harv.) } \\
\text { Guiry }\end{array}$ & 0.6 & 0.9 & 0.6 & & & 0.2 & 0.8 & 0.5 & ${ }^{1} 0.4 \pm 0.1 \mathrm{~ns}$ \\
\hline $\begin{array}{l}\text { Pterocladiella capillacea (Gmel.) } \\
\text { Santelices \& Hommers. }\end{array}$ & 0.5 & 0.1 & & & & 0.1 & 1.5 & 0.7 & ${ }^{2} 0.3 \pm 0.3^{* *}$ \\
\hline Cryptopleura crispa Kyl. & 0.3 & 0.2 & 0.1 & & & 0.3 & 1.6 & 0.2 & ${ }^{1} 0.3 \pm 0.2^{* * *}$ \\
\hline $\begin{array}{l}\text { Corallopbila eatoniana (Farl.) Cho, } \\
\text { Choi, Hansen \& Boo DeToni } \\
\text { and C. sinicola S. \& G. }\end{array}$ & 0.4 & 0.8 & 0.1 & & & 0.2 & 0.8 & & ${ }^{2} 0.3 \pm 0.2 \mathrm{~ns}$ \\
\hline $\begin{array}{l}\text { Other frondose algae species } \\
(n=19)\end{array}$ & 3.2 & 1.8 & 0.5 & 0.1 & & 1.7 & 2.3 & 0.7 & $1.4 \pm 1.1$ \\
\hline Total frondose algal cover & 7.8 & 5.4 & 2.0 & 0.3 & 0.2 & 2.2 & 9.5 & 5.3 & $5.1 \pm 3.5$ \\
\hline Total understory seaweed cover & 70.0 & 83.0 & 56.6 & 57.2 & 72.6 & 63.0 & 94.4 & 75.4 & $72.4 \pm 4.6$ \\
\hline
\end{tabular}

Note: Superscripts indicate data were transformed before ANOVA analyses ( $\left.{ }^{(}\right)$or were subjected to a randomization test following evaluation of variance ratios $\left({ }^{2}\right)$. All data sets subjected to randomization testing showed equal population dispersions based on the ratio of the largest to the smallest variance. Significant differences among sites are indicated by asterisks: ${ }^{\star}, P<0.05 ;{ }^{\star *}, P<0.01$;
${ }^{\star *}, P<0.001$.

"Sites located within intertidal MPAs designated between 1969 and 1972 are indicated by a $\dagger$.

cover (Tables 6, 7). No other taxon averaged more than $2 \%$ cover over the eight sites. The fleshy crust Pseudolithoderma nigra was the only species observed in all sampled quadrats and contributed mean cover ranging from $11.3 \%$ at Woods Cove to $50.8 \%$ at Thousand Steps. The next most abundant species was the coralline crust $P$. neofarlowii, which ranged from $10.2 \%$ (Thousand Steps) to 28.8\% (Dana Point) cover.
Cover of the three seaweed suprataxa and most seaweed populations varied significantly among sites (Tables 6,7) but not between autumn and spring ( $t$-tests; $P>0.05$ ). The most abundant component of the subcanopy community was the crustose algal group, which contributed cover ranging from $38.4 \%$ at Crystal Cove to $69.1 \%$ at Thousand Steps (Tables 6,7 ). The articulated coralline algal group was the second largest contributor 
to understory cover, averaging ca. $12 \%$ over all sites. Articulated coralline cover varied greatly among sites, ranging from only $0.8 \%$ at Victoria Beach to $40.6 \%$ at Monarch Bay (Tables 6, 7). A significant but weak negative correlation $(r=-0.20, P<0.05)$ was found between articulated coralline and crustose algal cover, suggesting that crustose algae became more abundant when cover of articulated corallines was reduced beneath the $S$. compressa canopy. The frondose algal group consisted of far more species (34) than any other seaweed group, but averaged only $4.6 \%$ cover per site. Frondose algal cover showed as much as a sevenfold difference among sites, with greatest cover at Monarch Bay. A positive correlation $(r=0.66, P<0.001)$ was found between articulated coralline and frondose algal cover, possibly because of the large number of small algal epiphytes growing on Corallina spp.

SESSILE INVERTEBRATES. The mean cover of the sessile invertebrate group ranged from 5.8 to $54.5 \%$ and varied significantly among sites (Tables 8,9 ) but not between sampling periods $(t=-0.438, P=0.68)$. Greatest cover was found at Woods Cove, whereas least cover occurred at Thousand Steps in the autumn and Shaw's Cove in the spring, both sites subjected to high sand influence (Tables 8, 9). A significant negative correlation $(r=-0.48, P<0.001)$ was found between sessile invertebrate and crustose algal cover, suggesting that these groups might compete for subcanopy space.

Of the 20 sessile macroinvertebrates, the four most abundant species contributed an average of $20.2 \%$ of the annualized understory cover. These were the sandy tubeworm Pbragmatopoma californica, the mussel Mytilus californianus, the acorn barnacles Cbthamalus fissus/C. dalli, and the sea anemone Antbopleura elegantissima (Tables 8, 9). Cover of $P$. californica varied widely among sites, ranging from $0.8 \%$ at Thousand Steps (autumn) to $26.3 \%$ (autumn) at Woods Cove (Table 9). During both assessment periods, Thousand Steps had low $P$. californica cover, high crustose algal cover, and high sand influence. High $P$. californica cover corresponded with low sand influence and high wave force at
Woods Cove and Treasure Island. Cover of Mytilus califormianus was greatest at Victoria Beach and Woods Cove, two of the more wave-exposed sites. Of the sessile macroinvertebrates, only Anthopleura elegantissima showed significant differences in cover $(t=$ $-2.557, P=0.04)$ between the two sampling periods.

mobile invertebrates. Mobile invertebrate densities in the understory habitat ranged from 125.4 to $637.7 \mathrm{~m}^{-2}$ (Tables 8,9 ). The three most abundant mobile invertebrates (Littorina scutulata, Lepidocbitona bartwegii, and Macclintockia scabra/Lottia conus) accounted for nearly $60 \%$ of all individuals. Only two other taxa (Lottia strigatella and Nuttallina fluxa/N. californica) averaged densities greater than $20 \mathrm{~m}^{-2}$ across the eight sites. Greatest mobile invertebrate densities occurred at Shaw's Cove in the autumn and Woods Cove in the spring, sites that supported high numbers of the periwinkle Littorina scutulata and the limpets Macclintockia scabra and Lottia conus. Lowest mobile invertebrate densities occurred at Thousand Steps in the autumn and Monarch Bay in the spring, both sites with low densities of $L$. scutulata. Densities of most mobile invertebrate populations varied significantly among sites (Tables 8,9 ) but not between assessment periods ( $t$-tests; $P>0.05)$. Limpet and chiton densities were least at Dana Point and Monarch Bay, the southernmost sites, and greatest at Crystal Cove and Woods Cove. Lower densities of limpets and chitons generally occurred at the more sandinfluenced sites. A weak, positive correlation $(r=0.30, P<0.05)$ was found between sessile invertebrate cover and limpet and chiton densities.

\section{Biological Diversity}

A total of 111 taxa was distinguished at the eight sites during the study, including 47 algae and 20 sessile and 44 mobile macroinvertebrates (see Appendix). An average of 27 macrophytes and sessile macroinvertebrates and 19 mobile macroinvertebrates was found across all sites. The number of macrophytes and sessile macroinvertebrates ranged 
TABLE 8

Mean Cover and Density for Abundant Sessile and Mobile Macroinvertebrates for Autumn 1995

\begin{tabular}{|c|c|c|c|c|c|c|c|c|c|}
\hline \multirow[b]{2}{*}{ Macroinvertebrate Taxa } & \multicolumn{8}{|c|}{ Sites $^{a}$} & \multirow[b]{2}{*}{ Mean $\pm \mathrm{SE}$} \\
\hline & $\mathrm{ICRC}$ & †SHW & WDS & VIC & TRI & THS & $\dagger \mathrm{MBY}$ & $\dagger \mathrm{DPT}$ & \\
\hline \multicolumn{10}{|l|}{ Sessile invertebrate cover } \\
\hline $\begin{array}{l}\text { Phragmatopoma californica (Fewkes, } \\
\text { 1889) }\end{array}$ & 21.5 & 3.3 & 21.6 & 9.0 & 22.0 & 1.7 & 14.4 & 6.6 & ${ }^{1} 12.5 \pm 3.0^{\star \star \star}$ \\
\hline Mytilus californianus Conrad, 1837 & 3.4 & 0.9 & 8.1 & 8.3 & 1.7 & 1.2 & 1.4 & 0.4 & $23.1 \pm 1.1^{* *}$ \\
\hline $\begin{array}{l}\text { Cbtbamalus fissus Darwin, } 1854 \text { and } \\
\text { C. dalli Pilsbry, } 1916\end{array}$ & 2.7 & 2.8 & 3.5 & 5.6 & 2.9 & 0.6 & 1.4 & 2.6 & $12.7 \pm 0.5 \mathrm{~ns}$ \\
\hline $\begin{array}{l}\text { Antbopleura elegantissima (Brandt, } \\
\text { 1835) }\end{array}$ & 3.8 & 1.6 & 0.7 & 2.3 & 0.7 & 2.0 & 1.2 & 0.9 & ${ }^{1} 1.6 \pm 0.4^{\star *}$ \\
\hline $\begin{array}{l}\text { Septifer bifurcatus (Conrad, 1837) } \\
\text { and Bracbidontes adamsianus } \\
\text { (Dunker, 1857) }\end{array}$ & 1.1 & 1.1 & 1.4 & 1.9 & 1.6 & 0.2 & 0.4 & 0.6 & ${ }^{1} 1.1 \pm 0.2 \mathrm{~ns}$ \\
\hline Tetraclita rubescens Darwin, 1854 & & 0.1 & 3.7 & 0.3 & 1.9 & & & & ${ }^{2} 0.7 \pm 0.6^{* * *}$ \\
\hline Balanus glandula Darwin, 1854 & 1.0 & 0.2 & 0.1 & 0.9 & 0.1 & 0.1 & & & ${ }^{2} 0.3 \pm 0.2 \mathrm{~ns}$ \\
\hline \multicolumn{10}{|l|}{ Other sessile invertebrates } \\
\hline (Number of taxa) & $(5)$ & $(6)$ & $(8)$ & (2) & (4) & $(0)$ & (6) & (1) & $(10)$ \\
\hline Combined cover & 1.6 & 1.3 & 1.5 & 0.4 & 0.6 & 0.1 & 1.6 & 0.1 & $1.2 \pm 0.3$ \\
\hline Total sessile invertebrate cover & 34.8 & 11.3 & 40.6 & 28.7 & 31.5 & 5.8 & 20.4 & 11.2 & $23.0 \pm 4.5$ \\
\hline \multicolumn{10}{|l|}{ Mobile invertebrate density (no. $\mathrm{m}^{-2}$ ) } \\
\hline Littorina scutulata Gould, 1849 & 50.0 & 285.5 & 130.1 & 214.1 & 150.7 & 8.0 & 17.3 & 70.7 & ${ }^{1} 115.8 \pm 34.7^{* * *}$ \\
\hline $\begin{array}{l}\text { Lepidochitona bartwegii (Carpenter, } \\
\text { 1855) }\end{array}$ & 62.7 & 86.7 & 42.7 & 26.7 & 42.0 & 40.0 & 52.0 & 50.7 & $50.4 \pm 6.4^{*}$ \\
\hline $\begin{array}{l}\text { Macclintockia scabra (Gould, 1864) } \\
\text { and Lottia conus (Test, 1945) }\end{array}$ & 32.7 & 49.4 & 19.3 & 38.7 & 97.4 & 16.7 & 44.0 & 17.3 & ${ }^{1} 39.4 \pm 9.4^{* * *}$ \\
\hline $\begin{array}{r}\text { Nuttallina fluxa (Carpenter, 1864) } \\
\text { and N. californica (Reeve, 1847) }\end{array}$ & 17.3 & 23.3 & 48.7 & 26.7 & 42.0 & 6.7 & 28.7 & 16.0 & $26.2 \pm 4.9^{\star \star}$ \\
\hline Lottia strigatella (Carpenter, 1864) & 75.4 & 22.0 & 27.3 & 15.3 & 34.0 & 17.3 & 12.7 & 2.7 & ${ }^{1} 25.8 \pm 7.8^{* * * *}$ \\
\hline Lottia pelta (Rathke, 1833) & 26.7 & 14.0 & 40.0 & 13.3 & 32.0 & 26.0 & 5.3 & 1.3 & ${ }^{\mathrm{I}} 19.8 \pm 4.8^{\star \star \star}$ \\
\hline $\begin{array}{l}\text { Acantbina spirata (de Blainville, } \\
\text { 1832) }\end{array}$ & 46.7 & 30.0 & 16.7 & 14.0 & 11.3 & 3.3 & 27.3 & 0.7 & ${ }^{1} 18.8 \pm 5.4^{* * *}$ \\
\hline Pagumus samuelis (Stimpson, 1857) & 14.7 & 31.3 & 1.3 & & 3.3 & & 12.7 & 4.0 & ${ }^{\mathrm{I}} 8.4 \pm 3.8^{* * *}$ \\
\hline Tegula gallina (Forbes, 1852) & 0.7 & 2.0 & & & & 3.3 & 2.0 & 33.4 & ${ }^{2} 5.2 \pm 4.0^{\star \star \star}$ \\
\hline $\begin{array}{l}\text { Nucella emarginata (Deshayes, } \\
\text { 1839) }\end{array}$ & 2.0 & 4.0 & 10.7 & 12.0 & 6.7 & & & & ${ }^{2} 4.4 \pm 1.7^{\star}$ \\
\hline Littorina keenae Rosewater, 1978 & & 6.0 & 8.7 & 0.7 & 4.7 & 0.7 & & 0.7 & ${ }^{2} 2.7 \pm 1.2 \mathrm{~ns}$ \\
\hline $\begin{array}{l}\text { Epitonium tinctum (Carpenter, } \\
\text { 1864) }\end{array}$ & 9.3 & & 1.3 & 1.3 & 0.7 & 1.3 & 4.0 & 1.3 & ${ }^{2} 2.4 \pm 1.1 \mathrm{~ns}$ \\
\hline Macron lividus (A. Adams, 1855) & 4.0 & 8.7 & 0.7 & & 6.0 & & & & ${ }^{2} 2.4 \pm 1.2^{\star *}$ \\
\hline Lottia limatula (Carpenter, 1864) & 1.3 & 8.0 & 1.3 & 3.3 & & 0.7 & & 1.3 & $22.0 \pm 0.9 \mathrm{~ns}$ \\
\hline Tegula eiseni Jordan, 1936 & 0.7 & 0.7 & & & & 0.7 & 11.3 & & ${ }^{2} 1.7 \pm 1.4^{\star \star *}$ \\
\hline Mopalia muscosa (Gould, 1846) & 2.0 & 0.7 & 0.7 & 4.7 & 2.7 & & & 0.7 & $1.4 \pm 0.6^{*}$ \\
\hline Conus califormicus Reeve, 1844 & 1.3 & & & & & & 7.3 & 1.3 & ${ }^{2} 1.2 \pm 0.9^{\star *}$ \\
\hline Pacbygrapsus crassipes Randall, 1839 & 2.7 & 1.3 & 1.3 & 0.7 & 1.3 & & 1.3 & & ${ }^{1} 1.1 \pm 0.3 \mathrm{~ns}$ \\
\hline Other mobile invertebrates & & & & & & & & & \\
\hline (Number of taxa) & (1) & (6) & (2) & $(5)$ & (4) & (1) & $(12)$ & (3) & (17) \\
\hline Combined densities & 1.4 & 8.0 & 1.4 & 10.2 & 2.9 & 0.7 & 15.6 & 7.4 & $5.9 \pm 1.9$ \\
\hline Total mobile invertebrate density & 351.6 & 581.6 & 352.2 & 381.7 & 437.7 & 125.4 & 241.5 & 209.5 & $335.1 \pm 50.4$ \\
\hline
\end{tabular}

Note: Superscript indicates data were transformed before ANOVA analyses $\left({ }^{1}\right)$ or were subjected to a randomization test following evaluation of variance ratios $\left({ }^{2}\right)$. Only the Mytilus califormianus cover data showed unequal population dispersions based on the ratio of the largest to the smallest variance so this randomization test result should be interpreted with caution. Significant differences among sites are indicated by asterisks: ${ }^{*}, P<0.05$; $^{* \star}, P<0.01$; ${ }^{* * *}, P<0.001$.

"Sites located within intertidal MPAs designated between 1969 and 1972 are indicated by a $f$. 
TABLE 9

Mean Cover and Density for Abundant Sessile and Mobile Macroinvertebrates for Spring 1996

\begin{tabular}{|c|c|c|c|c|c|c|c|c|c|}
\hline \multirow[b]{2}{*}{ Macroinvertebrate Taxa } & \multicolumn{8}{|c|}{ Sites $^{a}$} & \multirow[b]{2}{*}{ Mean $\pm S E$} \\
\hline & †CRC & $\dagger \mathrm{SHW}$ & WDS & VIC & TRI & THS & $\dagger M B Y$ & †DPT & \\
\hline \multicolumn{10}{|l|}{ Sessile invertebrate cover } \\
\hline $\begin{array}{l}\text { Phragmatopoma californica (Fewkes, } \\
1889 \text { ) }\end{array}$ & 11.9 & 4.3 & 26.3 & 14.2 & 19.7 & 0.8 & 15.8 & 7.8 & ${ }^{1} 12.6 \pm 2.9^{\star * *}$ \\
\hline $\begin{array}{l}\text { Chtbamnalus fussus Darwin, } 1854 \text { and } \\
\text { C. dalli Pilsbry }\end{array}$ & 3.1 & 1.1 & 7.5 & 1.0 & 0.6 & 1.4 & 1.0 & 5.9 & ${ }^{1} 2.7 \pm 0.9^{* * *}$ \\
\hline $\begin{array}{l}\text { Anthopleura elegantissima (Brandt, } \\
\text { 1835) }\end{array}$ & 5.3 & 1.4 & 3.2 & 2.6 & 1.1 & 3.4 & 0.3 & 3.4 & $2.6 \pm 0.6^{* * *}$ \\
\hline Mytilus californianus Conrad, 1837 & 1.4 & 0.5 & 7.0 & 2.8 & 0.8 & 3.6 & 1.1 & 2.7 & $2.5 \pm 0.7^{* * *}$ \\
\hline $\begin{array}{l}\text { Septifer bifurcatus (Conrad, 1837) and } \\
\text { Brachidontes adamsianus (Dunker, } \\
1857 \text { ) }\end{array}$ & 1.2 & 0.5 & 3.1 & 3.5 & 2.1 & 1.0 & 0.4 & 0.7 & ${ }^{\mathrm{I}} 1.5 \pm 0.4^{* * *}$ \\
\hline Tetraclita rubescens Darwin, 1854 & 0.1 & 0.3 & 4.2 & 0.8 & 1.6 & & 0.1 & 0.1 & ${ }^{2} 0.9 \pm 0.6^{\star \star \star \star}$ \\
\hline Pseudochama exogyra (Conrad, 1837) & 0.9 & 0.7 & 0.4 & 0.2 & 0.1 & 0.1 & 0.4 & 0.3 & $0.4 \pm 0.1^{* * *}$ \\
\hline Balanus glandula Darwin, 1854 & 0.4 & & 0.9 & & 0.1 & 0.2 & & 0.4 & ${ }^{2} 0.3 \pm 0.1^{*}$ \\
\hline \multicolumn{10}{|l|}{ Other sessile invertebrates } \\
\hline (Number of taxa) & $(6)$ & (5) & (6) & (3) & $(5)$ & $(0)$ & (5) & $(2)$ & $(10)$ \\
\hline Combined cover & 0.6 & 0.8 & 2.0 & 0.9 & 1.7 & 0.0 & 0.7 & 0.4 & $0.9 \pm 0.2$ \\
\hline Total sessile invertebrate cover & 24.9 & 9.5 & 54.5 & 25.8 & 27.7 & 10.5 & 19.6 & 21.5 & $24.3 \pm 4.9$ \\
\hline \multicolumn{10}{|l|}{ Mobile invertebrate density (no. $\mathrm{m}^{-2}$ ) } \\
\hline Littorina scutulata Gould, 1849 & 24.0 & 45.4 & 138.1 & 230.1 & 268.8 & 102.1 & 7.3 & 138.1 & ${ }^{1} 119.2 \pm 33.5^{* * *}$ \\
\hline $\begin{array}{l}\text { Lepidocbitona bartwegii (Carpenter, } \\
\text { 1855) }\end{array}$ & 83.4 & 44.7 & 68.0 & 58.0 & 54.0 & 44.0 & 20.7 & 54.0 & $53.4 \pm 6.5^{\star}$ \\
\hline $\begin{array}{l}\text { Macclintockia scabra (Gould, 1864) } \\
\text { and L. conus (Test, 1945) }\end{array}$ & 68.0 & 48.7 & 136.1 & 24.7 & 59.4 & 24.0 & 18.7 & 12.7 & ${ }^{1} 44.3 \pm 14.3^{* * *}$ \\
\hline Lottia strigatella (Carpenter, 1864) & 106.4 & 14.7 & 107.4 & 14.7 & 22.0 & 55.4 & 10.7 & 10.0 & ${ }^{1} 42.6 \pm 14.9^{* * *}$ \\
\hline $\begin{array}{l}\text { Nuttallina fluxa (Carpenter, 1864) } \\
\text { and } N \text {. californica (Reeve, 1847) }\end{array}$ & 26.7 & 27.3 & 82.7 & 10.7 & 60.0 & 17.3 & 18.7 & 14.0 & $32.2 \pm 9.0^{* * *}$ \\
\hline Lottia pelta (Rathke, 1833) & 28.0 & 13.3 & 41.4 & 24.0 & 22.0 & 14.0 & 3.3 & 6.0 & $19.0 \pm 4.4^{* *}$ \\
\hline Tegula gallina (Forbes, 1852) & 3.3 & 13.3 & & & & 6.0 & 24.7 & 86.0 & ${ }^{2} 16.7 \pm 12.1^{\star \star \star}$ \\
\hline Acantbina spirata (de Blainville, 1832) & 16.7 & 14.0 & 8.7 & 9.3 & 20.0 & 7.3 & 18.7 & 15.3 & $13.8 \pm 1.7 \mathrm{~ns}$ \\
\hline Pagurus samuelis (Stimpson, 1857) & 14.0 & 34.0 & 3.3 & & 44.0 & 0.7 & 6.0 & 2.0 & ${ }^{2} 13.0 \pm 6.1^{*}$ \\
\hline Nucella enarginata (Deshayes, 1839) & 0.7 & 1.3 & 12.7 & 0.7 & 11.3 & 15.3 & & 2.0 & $25.4 \pm 2.3^{* * *}$ \\
\hline Epitonium tinctum (Carpenter, 1864) & 13.3 & 2.0 & 1.3 & 8.7 & 0.7 & 6.7 & & 3.3 & $4.5 \pm 1.6 \mathrm{~ns}$ \\
\hline Lottia limatula (Carpenter, 1864) & 1.3 & 4.0 & 13.3 & 4.0 & 1.3 & & 4.7 & 6.0 & $4.3 \pm 1.4^{\star \star}$ \\
\hline Pachygrapsus crassipes Randall, 1839 & 4.7 & 0.7 & 11.3 & 6.0 & 6.0 & 1.3 & 0.7 & 0.7 & $13.9 \pm 1.4^{* * *}$ \\
\hline Tegula funebralis (A. Adams, 1855) & 8.0 & 1.3 & 0.7 & & & 4.0 & 1.3 & 6.7 & $12.8 \pm 1.1^{* *}$ \\
\hline Lottia spp. (juveniles) & & 2.7 & 6.0 & 2.0 & & & 9.3 & & $22.5 \pm 1.2 \mathrm{~ns}$ \\
\hline Conus californicus Reeve, 1844 & 4.0 & 0.7 & & & & 1.3 & 6.0 & 1.3 & ${ }^{2} 1.7 \pm 0.8 \mathrm{~ns}$ \\
\hline Macron lividus (A. Adams, 1855) & 6.0 & & 0.7 & & 5.3 & & & & $21.5 \pm 1.0^{*}$ \\
\hline Mopalia muscosa (Gould, 1846) & 4.0 & 2.7 & 0.7 & 1.3 & 0.7 & 2.0 & 0.7 & & ${ }^{1} 1.5 \pm 0.4 \mathrm{~ns}$ \\
\hline Tegutla eiseni Jordan, 1836 & & & & & 0.7 & 0.7 & 9.3 & 0.7 & ${ }^{2} 1.4 \pm 1.5^{* * *}$ \\
\hline Fissurella volcano Reeve, 1849 & 2.0 & 2.7 & 0.7 & & & & 2.0 & 0.7 & ${ }^{2} 1.0 \pm 0.3 \mathrm{~ns}$ \\
\hline Other mobile invertebrates & & & & & & & & & \\
\hline (Number of taxa) & (1) & (2) & (6) & (4) & (4) & $(0)$ & (5) & (4) & $(15)$ \\
\hline Combined densities & 1.3 & 2.0 & 4.7 & 5.3 & 3.3 & 0.0 & 4.0 & 4.0 & $3.1 \pm 0.6$ \\
\hline Total mobile invertebrate density & 415.5 & 277.5 & 637.7 & 399.5 & 579.6 & 302.2 & 166.8 & 363.5 & $392.8 \pm 54.9$ \\
\hline
\end{tabular}

Note: Superscript indicates data were transformed before ANOVA analyses $\left({ }^{1}\right)$ or were subjected to a randomization test following evaluation of variance ratios $\left({ }^{2}\right)$. All data sets subjected to randomization testing showed equal population dispersions based on the ratio of the largest to the smallest variance. Significant differences among sites are indicated by asterisks: ${ }^{*}, P<0.05 ;{ }^{*}, P<0.01$; ${ }^{* * *}, P<0.001$.

"Sites located within intertidal MPAs designated between 1969 and 1972 are indicated by a $\uparrow$. 
TABLE 10

Site Diversity Based on Macrophyte and Sessile Macroinvertebrate Cover and Mobile Macroinvertebrate Densities

\begin{tabular}{|c|c|c|c|c|c|c|}
\hline \multirow[b]{2}{*}{ Site ${ }^{n}$} & \multicolumn{3}{|c|}{$\mathrm{S}^{b}$} & \multicolumn{3}{|c|}{$H_{c}^{\prime \epsilon}$} \\
\hline & Autumn & Spring & Site Mean & Autumn & Spring & Site Mean \\
\hline \multicolumn{7}{|l|}{ Cover } \\
\hline †Crystal Cove & 40 & 37 & 38.5 & 2.39 & 2.20 & 2.30 \\
\hline Shaw's Cove & 24 & 31 & 27.5 & 1.49 & 2.01 & 1.75 \\
\hline Woods Cove & 34 & 28 & 31.0 & 2.15 & 2.39 & 2.27 \\
\hline Victoria Beach & 16 & 16 & 16.0 & 1.83 & 1.66 & 1.74 \\
\hline Treasure Island & 24 & 19 & 21.5 & 1.83 & 1.67 & 1.75 \\
\hline Thousand Steps & 25 & 26 & 25.5 & 1.34 & 1.57 & 1.46 \\
\hline tMonarch Bay & 30 & 32 & 31.0 & 2.08 & 2.10 & 2.09 \\
\hline Dana Point & 26 & 27 & 26.5 & 1.77 & 2.04 & 1.90 \\
\hline \multicolumn{7}{|l|}{ Density } \\
\hline †Crystal Cove & 18 & 19 & 18.5 & 2.21 & 2.23 & 2.22 \\
\hline HShaw's Cove & 22 & 20 & 21.0 & 1.83 & 2.34 & 2.08 \\
\hline Woods Cove & 17 & 23 & 20.0 & 1.98 & 2.09 & 2.04 \\
\hline Victoria Beach & 18 & 17 & 17.5 & 1.67 & 1.57 & 1.62 \\
\hline Treasure Island & 18 & 19 & 18.5 & 1.94 & 1.85 & 1.90 \\
\hline Thousand Steps & 13 & 16 & 14.5 & 1.92 & 2.04 & 1.98 \\
\hline †Monarch Bay & 25 & 22 & 23.5 & 2.43 & 2.59 & 2.51 \\
\hline Dana Point & 17 & 21 & 19.0 & 1.87 & 1.90 & 1.89 \\
\hline
\end{tabular}

"Sites located within intertidal MPAs designated between 1969 and 1972 are indicated by a $\uparrow$.

${ }^{b}$ Number of species.

'Shannon's Diversity Index.

from an average of 16 at Victoria Beach to 38.5 at Crystal Cove; the number of mobile macroinvertebrates ranged from 14.5 (Thousand Steps) to 23.5 (Monarch Bay) (Table $10) . H^{\prime}$ diversity values averaged 1.91 (macrophytes and sessile macroinvertebrates) and 2.03 (mobile macroinvertebrates). Highest $H^{\prime}$ diversity generally occurred at sites having the greatest richness.

\section{Relationships between Biological Data and Environmental Parameters}

QUADRAT-SCALE PATTERNS. Quadrats located near the upper reaches of the Silvetia compressa assemblage had higher abundances of sessile invertebrates, as supported by the very weak positive but significant correlation between sessile invertebrate cover and relative vertical tidal position $(r=0.18, P<$ $0.05)$. In contrast, significant, but weak, negative correlations were found between tidal position and articulated coralline $(r=$ $-0.35, P<0.001)$ and frondose $(r=-0.41$, $P<0.001$ ) algal cover. Thus, the upper boundaries of the assemblage were generally characterized by higher cover of sessile invertebrates, particularly acorn barnacles, whereas the lower portions supported greater cover of erect seaweeds.

Sand influence correlated with the abundances of understory algae and invertebrates. Quadrats with less sand had higher amounts of sessile invertebrate cover $(r=-0.50$, $P<0.001)$ and greater limpet and chiton densities $(r=-0.45, P<0.001)$. In contrast, significant, but weak, positive correlations were found between sand cover and crustose $(r=0.28, P<0.01)$, articulated coralline $(r=0.22, P<0.01)$, and frondose $(r=0.48$, $P<0.001)$ algal cover.

SITE-SCALE PATTERNS. Diversity values failed to show significant correlations with any measured environmental variable, and cluster analysis and MDS results failed to produce clear site patterns (Figures 2, 3). Generally, the autumn and spring site data were more closely associated with each other than with other sites, and long-standing MPAs did not consistently form groups. 

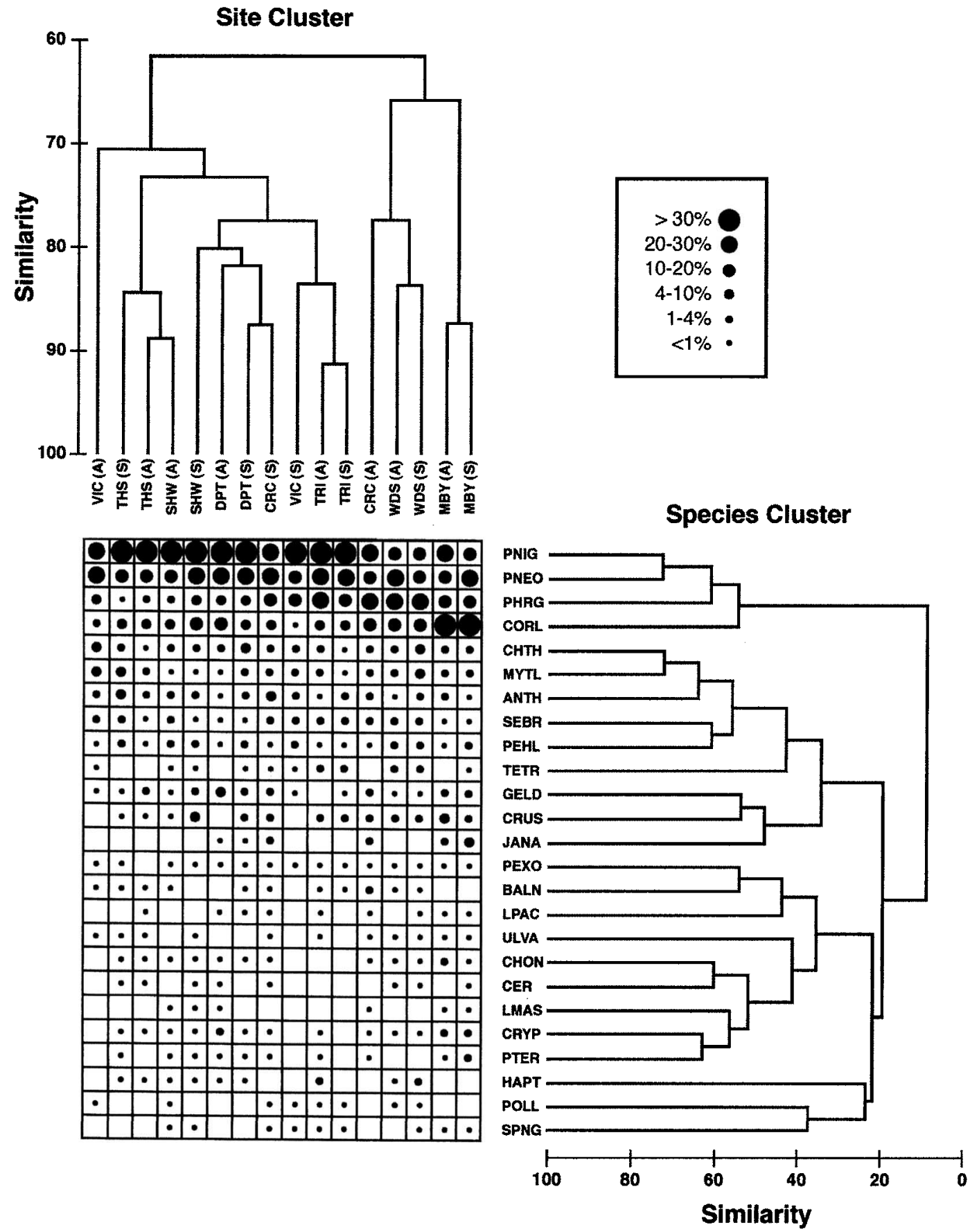

Figure 2. Results of hierarchical cluster analysis. See Table 1 for site abbreviations. A, autumn; S, spring. Species abbreviations: Macroinvertebrates: ANTH, Anthopleura elegantissima; BALN, Balanus glandula; CHTH, Cbthamalus dalli and C. fissus; MYTL, Mytilus californianus; PHRG, Pbragmatopoma californica; PEXO, Pseudochama exogyra; POLL, Pollicipes polymerus; SEBR, Septifer bifurcatus and Bracbidontes adamsianus; TETR, Tetraclita rubescens. Seaweeds: CHON, Chondracantbus canaliculatus; CORL, Corallina spp.; CRUS, unidentified crustose coralline; CRYP, Cryptopleura crispa; GELD, Gelidium coulteri and $G$. pusillum; HAPT, Hapteropbycus canaliculatus; JANA, Fania crassa; LMAS, Laurencia masoni; LPAC, Laurencia spp.; PEHL, Peyssonneliaceae and Hildenbrandiaceae fleshy crusts; PNIG, Pseudolithoderma nigra; PNEO, Pseudolithophyllum neofarlowii; PTER, Pterocladiella capillacea; CER, Ceramintm spp.; ULVA, Ulva californica; SPNG, unidentified sponge. 
However, all sampled communities were very similar to one another, with most clustering together at greater than $80 \%$ similarity (Figure 2). Moreover, based on SIMPER analyses, the abundances of only four taxa (Pseudolithoderma nigra, Pseudolithophyllum neofarlowii, Pbragmatopoma californica, and Corallina spp.) contributed between 80.1 and $91.4 \%$ of the average within-group similarity and also were largely responsible for the dissimilarity between clusters. MDS analyses produced results similar to those obtained from cluster analysis (Figure 3). The more wave-exposed, least sand-influenced, and also more heavily human-influenced sites (Victoria Beach, Woods Cove, and Treasure Island) located relatively close together in both MDS ordinations and showed greatest separation from Monarch Bay. Although dominated by $P$. nigra, $P$. neofarlowii, $P$. californica, and Corallina spp., these sites had the highest cover of macroinvertebrates and generally the lowest cover of frondose algae.

ANOSIM tests failed to reveal significant differences between long-standing and recently established MPAs based on macrophyte and macroinvertebrate cover and mobile invertebrate densities (Table 11). Similar to results of population-level analyses (Tables 6-9), significant differences in community structure were detected among sites within both MPA groups. ANOSIM tests found significant differences in community composition between autumn and spring, with the exception of cover-based comparisons within the recently established MPA group.

\section{DISCUSSION}

Communities dominated by the rockweed Silvetia compressa commonly occur throughout southern California in upper, midshore habitats receiving protection from wave exposure (Ricketts et al. 1985, Murray and Bray 1993). For example, S. compressa (as $P$. fastigiata) ranked third in mean organic dry biomass among all intertidal seaweeds and macroinvertebrates sampled on southern California shores by Littler and colleagues in the 1970s (Littler 1980a,b, Littler et al. 1991). Yet, despite its common occurrence on southern California shores, little is known about variation in species composition and abundances in the $S$. compressa understory assemblage, either at an individual site or among sites within a region.

\section{Temporal Variation in S. compressa Cover}

Cover of Silvetia compressa was consistently higher during autumn and lower during spring at our study sites. Similar summer/ autumn peaks in abundance have been observed in southern (Gunnill 1980, Hill 1980) and central (Horn et al. 1983) California for $S$. compressa, and in southern California for the closely related fucoid Hesperopbycus californicus (as H. barveyanus [Oates 1981]). This pattern corresponds with a previous report (Littler et al. 1991) that the standing stocks of larger seaweeds grow and accumulate throughout the summer in southern California and decline through winter when desiccation stress corresponds with afternoon low tides. In the North Atlantic, intertidal fucoids (mostly Fucus spp.) also have been reported to grow at highest rates (Mathieson et al. 1976, Niemeck and Mathieson 1976, Sideman and Mathieson 1983, Mathieson 1989) and to reach greatest cover (Printz 1950, Hjohlman 1990, Gunnarsson and Ingólfsson 1995) from spring to late summer/ early autumn. Generally, seasonal increases in the growth rates and standing stocks of these upper-shore fucoids have been attributed to temporal changes in abiotic factors such as light, temperature, and the availability of nutrients, whereas decreases have been thought to be due to winter storms, desiccating conditions during tidal emersion, sediment inundation, and decreases in nutrients (Mathieson et al. 1976, Schonbeck and Norton 1978, Seapy and Littler 1982, Gunnill 1985, Hawkins and Hartnoll 1985, Gunnarsson and Ingólfsson 1995, Williams 1996).

\section{Species Composition and Diversity in the Subcanopy Assemblage}

SEAWEED. About $25 \%$ of the recorded seaweed species occurred in the Silvetia compressa understory assemblage at all eight sites, 


\section{MDS Plots}

Cover

Density

\begin{tabular}{|c|c|}
\hline $\begin{array}{l}\text { IHSA } \\
\text { Wa }\end{array}$ & 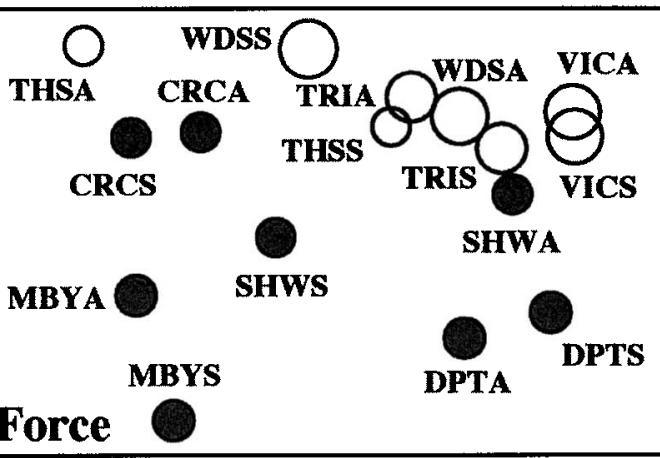 \\
\hline $\begin{array}{c}\text { OWDSS } \\
\text { OWDSA } \\
\text { CRCA }\end{array}$ & 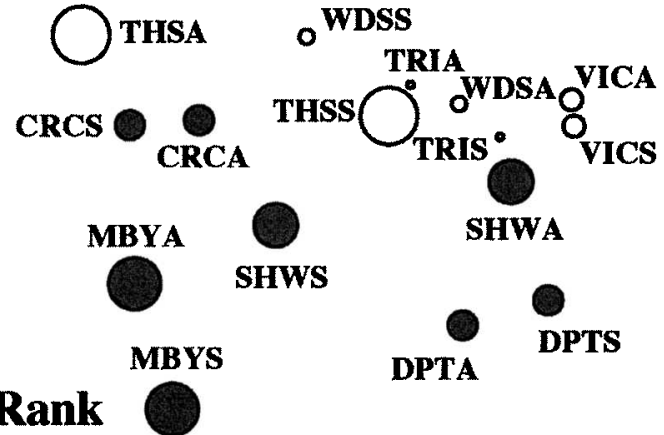 \\
\hline 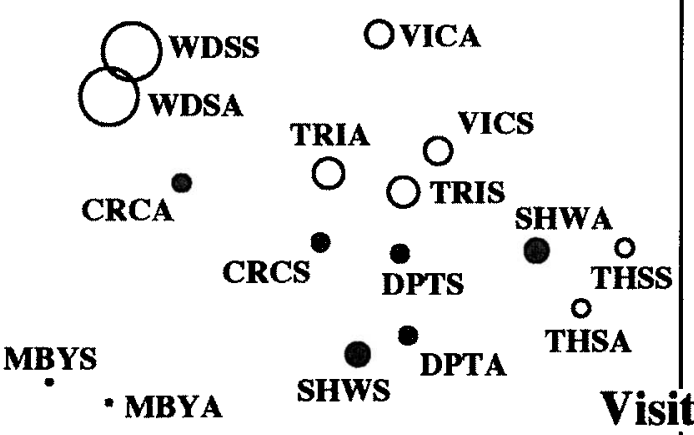 & 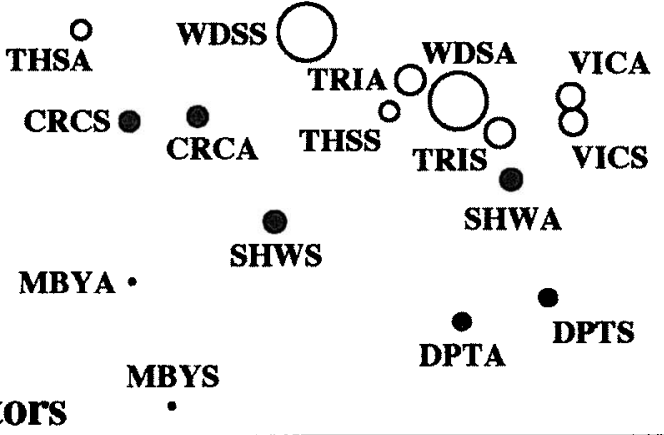 \\
\hline
\end{tabular}

FIGURE 3. Results of MDS ordinations based on cover of seaweeds and sessile macroinvertebrates and densities of mobile macroinvertebrates. Size of circle representing sites indicates relative magnitude of the indicated environmental variable. Depicted are wave force as the mean maximum wave force measured for each site over two seasons, sand influence as site ranks, and visitor use as the mean numbers of visitors per $10 \mathrm{~m}$ of site shoreline. See text for details of analysis, Table 1 for site abbreviations (with addition of A for autumn or S for spring), and Tables 2-4 for measured environmental parameters. MDS analyses based on untransformed cover data and standardized density data using all sampled taxa. Stress $=0.07$ Cover MDS; 0.10 Density MDS. 
TABLE 11

Summary of ANOSIM Test Results of Differences in Multivariate Structure

\begin{tabular}{|c|c|c|}
\hline Test & $\mathrm{R}$ & $P$ \\
\hline \multicolumn{3}{|c|}{ Longstanding MPAs versus recently established MPAs } \\
\hline \multicolumn{3}{|c|}{ Macrophyte and macroinvertebrate cover } \\
\hline Autumn & 0.094 & 0.257 \\
\hline Spring & 0.125 & 0.143 \\
\hline \multicolumn{3}{|c|}{ Mobile macroinvertebrate densities } \\
\hline Autumn & 0.087 & 0.200 \\
\hline Spring & 0.302 & 0.086 \\
\hline \multicolumn{3}{|c|}{ Site and season tests: Longstanding MPAs } \\
\hline \multicolumn{3}{|c|}{ Macrophyte and macroinvetebrate cover } \\
\hline Site & 0.342 & $<0.001^{\star * *}$ \\
\hline Season & 0.104 & $<0.001^{* * *}$ \\
\hline \multicolumn{3}{|c|}{ Mobile macroinvertebrate densities } \\
\hline Site & 0.396 & $<0.001^{* * *}$ \\
\hline Season & 0.167 & $<0.001^{* * *}$ \\
\hline \multicolumn{3}{|c|}{ Site and season tests: Recently established MPAs } \\
\hline \multicolumn{3}{|c|}{ Macrophyte and macroinvetebrate cover } \\
\hline Site & 0.393 & $<0.001^{* * *}$ \\
\hline Season & 0.033 & 0.144 \\
\hline \multicolumn{3}{|c|}{ Mobile macroinvertebrate densities } \\
\hline Site & 0.441 & $<0.001^{* * *}$ \\
\hline Season & 0.214 & $<0.001^{* * *}$ \\
\hline
\end{tabular}

Note; ANOSIM tests performed on untransformed cover and standardized density data for all sampled taxa. MPA tests based on a one-way ANOSIM model. Site and season tests based on a two-way crossed ANOSIM model. Significant test results are indicated by asterisks: ${ }^{* * *}, P<0.001$.

but only a few of these species dominated abundances at all sites. High cover of crustose algae beneath $S$. compressa fronds has been reported previously for rockweed communities in southern (Hill 1980) and central (Johnson and Brawley 1998) California. Crustose algae also characterize understory assemblages in intertidal rockweed communities elsewhere (Lewis 1964, Munda and Markham 1982) and appear to be dependent on canopy protection because they often fail to persist after canopy removal (Hawkins and Hartnoll 1985).

Although patchy and variable in abundance among sites, articulated coralline algae were commonly encountered in the Silvetia compressa understory assemblage. It is interesting that articulated corallines were more than three times more abundant at Monarch Bay, the site with the largest $S$. compress $a$ thalli and with greatest spatial separation between individual plants. Both crustose and articulated coralline algae are resistant to distur- bance from wave action (Norton et al. 1982), sand scour (Stewart 1982, 1983, Littler et al. 1983), and grazers (Littler and Littler 1980, Van Alstyne 1990) and may be favored beneath fucoid canopies where grazers are abundant (Hartnoll and Hawkins 1980, 1985, Schonbeck and Norton 1980, Hawkins and Hartnoll 1983b) and whiplash disturbance is high (Norton 1986, Raffaelli and Hawkins 1996). More susceptible to sources of disturbance (Littler and Littler 1980), frondose algal cover was much less abundant in the understory assemblage, and species occurred mostly as small saxicolous thalli or as epiphytes on articulated corallines.

macroinvertebrates. Total cover of sessile macroinvertebrates varied greatly among sites mostly due to differences in the abundance of Pbragmatopoma californica, a tube-building polychaete. It is interesting that $P$. californica, a species that requires sand to build its tubes (Morris et al. 1980), was less abundant at the more sand-influenced of our 
study sites. Compared with qualitative observations made on patches of adjacent rock, barnacle abundances were generally reduced beneath the $S$. compressa canopy, perhaps because of frond whiplash or the barrier presented by fronds to settlement of barnacle larvae (Stephenson 1939, Pyefinch 1943, Burrows and Lodge 1950, B. A. Menge 1976, Hawkins 1981, 1983, Hawkins and Hartnoll 1983a,b, Hartnoll and Hawkins 1985). Barnacle cover was highest at the upper edges of the $S$. compressa assemblage where fronds were thinnest, grazers least abundant, and periods of tidal emersion and desiccation stress were greatest. Interactions between fucoids, barnacles, and mobile invertebrate grazers are known to vary across environmental gradients and to account for spatial patchiness in rockweed communities (Hawkins and Hartnoll 1983b, 1985).

Mobile invertebrates in the understory assemblage primarily consisted of limpets, chitons, and littorines. Limpets aggregate under larger fucoid clumps (Hartnoll and Hawkins 1980, 1985, Schonbeck and Norton 1980, Hawkins and Hartnoll 1983b) and graze sporelings, reducing algal abundance. Littorines (Littorina keenae and L. scutulata) were the most abundant of the mobile macroinvertebrates in the understory assemblage but have much smaller body sizes than the North Atlantic Littorina littorea, which is an important grazer of understory algae in North Atlantic fucoid communities (J. L. Menge 1975, Lubchenco 1980, 1983, Barker and Chapman 1990, Norton et al. 1990).

The chiton Lepidochitona bartwegii and the limpet Lottia pelta were consistently found at our study sites in association with $\dot{S}$. compressa fronds but were rarely observed at similar tidal elevations outside the rockweed canopy. In a previous study, L. hartwegii and $L$. pelta disappeared in quadrats after the removal of S. compressa fronds (Hill 1980). Thus, it appears that these species are strongly dependent on the $S$. compressa canopy to persist at this tidal level on southern California shores. Both species are believed to feed on S. compressa and other noncalcified algae (Connor 1976, Morris et al. 1980). Limpets and chi- tons can reduce the abundances of erect, frondose algae beneath rockweed canopies (Lubchenco and Menge 1978), but the impact of these species and the smaller littorines on the structure of $S$. compressa understory communities is unknown.

Biological Diversity. The subcanopy assemblages at the eight study sites consistently exhibited relatively low $H^{\prime}$ diversity. In contrast, the total number of seaweed and macroinvertebrate species found in this community at most sites was high. These findings support other comparable assessments of diversity in the southern California Silvetia compressa understory assemblage based on subcanopy macroalgal and macroinvertebrate populations (Hill 1980). Observations at our study sites, however, indicate that most species in the understory assemblage also occur outside the influence of $S$. compressa canopies where they occur in greater abundance. This corresponds with Gunnill's (1982) observation that only two of many species of small fauna occurred exclusively in association with S. compressa fronds.

\section{Temporal Variation in the Subcanopy Assemblage}

Temporal (autumn/spring) variation in species composition and in the abundances of most populations was low in the Silvetia compressa understory assemblage. However, seasonal differences in community structure were generally detectable by ANOSIM tests. Previous research on populations associated with fucacean rockweeds has revealed low levels of seasonality (Oates 1981, Munda and Markham 1982, Josselyn and West 1985), and seasonal variation of most southern California intertidal populations is thought to be low (Littler 1980a,b, Foster et al. 1988, Littler et al. 1991, Murray and Bray 1993) and usually less than variation due to local or sitespecific conditions (Murray and Bray 1993). However, considerable seasonal variation in subcanopy algal abundances has been found on colder New England shores where environmental conditions vary much more greatly over the year (Mathieson et al. 1991). 
Site-Scale Variation in the Subcanopy Assemblage

Although spatial variation on rocky shores has received much attention, few studies have described patterns of horizontal variation over short geographic distances (Foster et al. 1988). Most previous work has focused on vertical distributions and localized patchiness within sites (Mathieson 1979, Underwood 1981, Bourget et al. 1994, Underwood and Chapman 1996, Johnson et al. 1997) or effects of disturbances across sites resulting, for example, from wave exposure (Dayton 1975, McQuaid and Branch 1985, Kilar and McLachlan 1989), sewage outfalls (Littler and Murray 1975, 1978), or oil spills (De Vogelaere and Foster 1994, Smith and Simpson 1995).

Sessile invertebrate cover and mobile invertebrate densities were generally greatest at Woods Cove, Treasure Island, and Victoria Beach, the most wave-exposed of our study sites. Suspension-feeding sessile invertebrates reach greater abundances at wave-exposed sites (McQuaid et al. 1985), and several studies have described changes in species abundances and the trophic structure of intertidal communities across wave-exposure gradients (Lewis 1968, McQuaid et al. 1985, Ricketts et al. 1985, Foster et al. 1988). This suggests that even small variations in wave exposure, measured over scales of a half to several kilometers, might influence the structure of the $S$. compressa assemblage.

Sand disturbance, both within and across sites, can vary considerably over both short (days to weeks) (McQuaid and Dower 1990, Trowbridge 1996) and long (seasonal) (Markham 1973, Daly and Mathieson 1977, Stewart 1983) temporal scales. Crustose algae were found in abundance in the subcanopy assemblage at all sites, including those most influenced by sand. Greatest cover of articulated coralline algae and lower abundances of sessile and mobile invertebrates generally occurred at the more sand-influenced sites. Crustose algae are known to attain high abundances in intertidal habitats subjected to sand inundation (Littler et al. 1983, Murray and Littler 1984, Murray and Bray 1993, Dethier 1994). Articulated coralline algae also can survive extended periods of sand burial (Littler et al. 1983, Littler and Kauker 1984, Murray and Bray 1993) and often trap and hold large quantities of sediment (Stewart 1983, McQuaid and Dower 1990, Whorff et al. 1995). Sand has been reported to correspond with reduced abundances of barnacles, mussels, and limpets (Seapy and Littler 1982, Littler et al. 1983).

Grazers in temperate fucoid assemblages can have patchy distributions over spatial gradients (reviewed by Hawkins and Hartnoll $1983 b$ ) and can alter algal and invertebrate populations by grazing or removing potential competitors (Dethier 1981, 1994). Grazers were abundant at Victoria Beach and Treasure Island, two sites with high wave exposure, low sand influence, and greater abundances of sessile invertebrates.

Most of our sites are subjected to high human visitation, and the foot traffic and collecting and exploratory activities of these human visitors are known to impact intertidal populations on these shores (Murray and Denis 1997, Murray 1998, Murray et al. 1999, Denis and Murray 2001) and elsewhere (Addessi 1994, Brosnan and Crumrine 1994, Keough and Quinn 1998, Schiel and Taylor 1999). Levels of human visitation vary among our sites (Murray 1998, Murray et al. 1999; Table 2), but a strong relationship between the density of human use and species abundance patterns could not be detected. Moreover, ANOSIM tests revealed that $S$. compressa subcanopy community structure did not vary significantly between historical MPAs and sites only recently receiving MPA protection, indicating that MPA designation has had little effect on the structures of the $S$. compressa understory communities at our study sites.

\section{Implications for Among-Site Comparative Studies}

Much of the variation in species abundances over local or regional spatial scales has been attributed to differences in environmental 
conditions such as wave exposure, water motion, disturbance, microhabitat variation, and to biological interactions (reviewed by Foster et al. 1988). We found high similarity among sites (Figure 2), suggesting relatively little structural variation in the Silvetia compressa assemblage. Nevertheless, using ANOSIM tests we were able to detect significant differences in multivariate structure in all comparisons of individual sites. In addition, significant differences in the cover or density of many of the more abundant populations also were found among sites using univariate procedures. Oceanographic conditions, such as sea temperature and salinity, were highly similar among sites. Consequently, amongsite differences were likely due to site-specific disturbance histories, biological features (e.g., predation and grazing, recruitment), and what appear to be relatively small variations in environmental conditions (e.g., wave force, sand influence, habitat topography) (reviewed by Foster et al. 1988, Murray and Bray 1993). However, we were able to establish only weak relationships between variations in the abundances of subcanopy species or in the structure of the subcanopy assemblage and hypothetically important environmental or biotic factors that differed across study sites. These results for the relatively simple $S$. compressa understory assemblage underscore the difficulties in establishing post hoc relationships between environmental patterns and variations in species abundances in rocky intertidal habitats. They also suggest the importance of localized and stochastic disturbance histories in generating variation among populations and communities exposed to highly similar ocean conditions, even over distances of only a few kilometers.

\section{ACKNOWLEDGMENTS}

We acknowledge the field assistance of many individuals, but especially Laurie Len, Oscar Rivas, Janine Kido, Shana Heid Meulmester, and Teri Gibson Denis. We also thank Jill Moeller, Marin McDonald, Jerry Newberry, Michael Vo, Julie Goodson, and Michelle Priest for assistance with the deployment of the wave force meters. We are grateful for the work of two anonymous reviewers who provided suggestions on the manuscript, and to Kelly Donovan who prepared the figures. We also thank D. Pryor, H. Helling, the Monarch Bay Community and Beach Club, and Patti Schooley for facilitating access to our study sites. K. R. Clarke provided advice for performing and interpreting statistical tests executed with PRIMER. S.A.S. was a University of Southern California Sea Grant Trainee during this study. Elements of this research also were supported by the Departmental Association Council and the Department of Biological Science at California State University, Fullerton. The views expressed herein do not necessarily reflect the views of NOAA, the Minerals Management Service, or any of their subagencies. The U.S. government is authorized to reproduce and distribute copies of this paper for governmental purposes.

\section{Literature Cited}

Abbott, I. A., and G. J. Hollenberg. 1976. Marine algae of California. Stanford University Press, Stanford, California.

Addessi, L. 1994. Human disturbance and long term changes on a rocky intertidal community. Ecol. Appl. 4:786-797.

Barker, S. M., and A. R. O. Chapman. 1990. Feeding preferences of periwinkles among four species of Fucus. Mar. Biol. (Berl.) 106:113-118.

Bell, E. C., and M. W. Denny. 1994. Quantifying "wave exposure": A simple device for recording maximum velocity and results of its use at several field sites. J. Exp. Mar. Biol. Ecol. 181:9-29.

Bourget, E., J. DeGuise, and G. Daigle. 1994. Scales of substratum heterogeneity, structural complexity, and the early establishment of a marine epibenthic community. J. Exp. Mar. Biol. Ecol. 181:31-51.

Bray, J. R., and J. T. Curtis. 1957. An ordination of the upland forest communities of southern Wisconsin. Ecol. Monogr. 27:325-349.

Brophy, T. C., and S. N. Murray. 1989. Field and culture studies of a population of Endarachne binghamiae (Phaeophyta) from southern California. J. Phycol. 25:6-15. 
Brosnan, D. M., and L. L. Crumrine. 1994. Effects of human trampling on marine rocky shore communities. J. Exp. Mar. Biol. Ecol. 177:79-97.

Burrows, E. M., and S. M. Lodge. 1950. Note on the inter-relationships of Patella, Balanus, and Fucus on a semi-exposed coast. Rep. Mar. Biol. Stn. Port Erin. 62:30-34.

Chapman, A. R. O. 1995. Functional ecology of fucoid algae: Twenty-three years of progress. Phycologia 34:1-32.

Chapman, A. R. O., and C. R. Johnson. 1990. Disturbance and organization of macroalgal assemblages in the Northwest Atlantic. Hydrobiologia 192:77-121.

Clarke, K. R., and R. N. Gorley. 2001. PRIMER (Plymouth Routines in Multivariate Ecological Research), version 5. User manual/tutorial. PRIMER-E Ltd., Plymouth Marine Laboratory, Plymouth.

Clarke, K. R., and R. H. Green. 1988. Statistical design and analysis for a 'biological effects' study. Mar. Ecol. Prog. Ser. 46:213-226.

Clarke, K. R., and R. M. Warwick. 2001. Change in marine communities: An approach to statistical analysis and interpretation. 2nd ed. PRIMER-E Ltd., Plymouth Marine Laboratory, Plymouth.

Connor, M. S. 1976. Niche apportionment among the chitons Cyanoplax bartwegii and Mopalia muscosa and the limpets Collisella limatula and Collisella pelta under the brown alga Pelvetia fastigiata. Veliger 18 (Suppl.): 9-17.

Daly, M. A., and A. C. Mathieson. 1977. The effects of sand movement on intertidal seaweeds and selected invertebrates at Bound Rock, New Hampshire, U.S.A. Mar. Biol. (Berl.) 43:45-55.

Dayton, P. K. 1975. Experimental evaluation of ecological dominance in a rocky intertidal algal community. Ecol. Monogr. 45:137-159.

Denis, T. G., and S. N. Murray. 2001. Among-site variation in the effects of trampling disturbance on Silvetia compressa (O. Fucales) populations. J. Phycol. 37 (Suppl.): 16.

Dethier, M. N. 1981. Heteromorphic algal life histories: The seasonal pattern and response to herbivory of the brown crust, Ralfsia californica. Oecologia (Berl.) 49:333-339.

1994. The ecology of intertidal algal crusts: Variation within a functional group. J. Exp. Mar. Biol. Ecol. 177:37-71.

De Vogelaere, A. P., and M. S. Foster. 1994. Damage and recovery in intertidal Fucus gardneri assemblages following the 'Exxon Valdez' oil spill. Mar. Ecol. Prog. Ser. 106:263-271.

Foster, M. S., A. P. De Vogelaere, C. Harrold, J. S. Pearse, and A. B. Thum. 1988. Causes of spatial and temporal patterns in rocky intertidal communities of central and northern California. Calif. Acad. Sci. Mem. 9:1-45.

Foster, M. S., C. Harrold, and D. D. Hardin. $1991 a$. Point vs. photo quadrat estimates of the cover of sessile marine organisms. J. Exp. Mar. Biol. Ecol. 146:193-203.

Foster, M. S., A. P. De Vogelaere, J. S. Oliver, J. S. Pearse, and C. Harrold. $1991 \mathrm{~b}$. Open coast intertidal and shallow subtidal ecosystems of the Northeast Pacific. Pages 235-272 in A. C. Mathieson and P. H. Nienhuis, eds. Ecosystems of the world. Vol. 24. Intertidal and littoral ecosystems. Elsevier, Amsterdam.

Green, R. H. 1979. Sampling design and statistical methods for environmental biologists. John Wiley \& Sons, New York.

Gunnarsson, K., and A. Ingólfsson. 1995. Seasonal changes in the abundance of intertidal algae in southwestern Iceland. Bot. Mar. 38:69-77.

Gunnill, F. C. 1980. Demography of the intertidal brown alga Pelvetia fastigiata in southern California, U.S.A. Mar. Biol. (Berl.) 59:169-179.

. 1982. Effects of plant size and distribution on the numbers of invertebrate species and individuals inhabiting the brown alga Pelvetia fastigiata. Mar. Biol. (Berl.) 69:263-280.

- 1983. Seasonal variation in the invertebrate faunas of Pelvetia fastigiata (Fucaceae): Effects of plant size and distribution. Mar. Biol. (Berl.) 73:115-130.

-1984. Differing distributions of potentially competing amphipods, copepods 
and gastropods among specimens of the intertidal alga Pelvetia fastigiata. Mar. Biol. (Berl.) 82:277-291.

1985. Growth, morphology, and microherbivore faunas of Pelvetia fastigiata (Phaeophyceae, Fucaceae) at La Jolla, California, U.S.A. Bot. Mar. 28:187-199.

Hartnoll, R. G., and S. J. Hawkins. 1980. Monitoring rocky-shore communities: A critical look at spatial and temporal variation. Helgol. Meeresunters. 33:484-494.

1985. Patchiness and fluctuations on moderately exposed rocky shores. Ophelia 24:53-63.

Hawkins, S. J. 1981. The influence of Patella grazing on the fucoid/barnacle mosaic on moderately exposed rocky shores. Kiel. Meeresforsch. 5:537-543.

- 1983. Interactions of Patella and macroalgae with settling Semibalanus balanoides (L.) J. Mar. Biol. Assoc. U.K. 71:55-72.

Hawkins, S. J., and R. G. Hartnoll. 1983a. Changes in a rocky shore community: An evaluation of monitoring. Mar. Environ. Res. 9:131-181. - 1983b. Grazing of intertidal algae by marine invertebrates. Oceanogr. Mar. Biol. Annu. Rev. 21:195-282.

1985. Factors determining the upper limits of intertidal canopy-forming algae. Mar. Ecol. Prog. Ser. 20:265-271.

Hickey, B. M. 1993. Physical oceanography. Pages 19-70 in M. D. Dailey, D. J. Reish, and J. W. Anderson, eds. Ecology of the Southern California Bight: A synthesis and interpretation. University of California Press, Berkeley.

Hill, M. L. 1980. Structure, organization, and persistence of the Pelvetia fastigiata (Phaeophyceae: Fucales) community on a rocky intertidal shoreline at Dana Point, Orange County, California. M.A. thesis, California State University, Fullerton.

Hjohlman, S. 1990. Initial succession samt säsongsvariation på Ascophyllum nodosumdominerad klippstrand. Ett fëltexperiment från Hordaland. Cand. Scient. thesis, Institutt for Fiskeri-og Marinbiologi, Universitetet I Bergen.

Horn, M. H., S. N. Murray, and R. R. Seapy. 1983. Seasonal structure of a cen- tral California rocky intertidal community in relation to environmental variations. Bull. South. Calif. Acad. Sci. 82:79-94.

Johnson, L. E., and S. H. Brawley. 1998. Dispersal and recruitment of a canopyforming intertidal alga: The relative roles of propagule availability and postsettlement processes. Oecologia (Berl.) 117:517-526.

Johnson, M. P., M. T. Burrows, R. G. Hartnoll, and S. J. Hawkins. 1997. Spatial structure on moderately exposed rocky shores: Patch scales and the interactions between limpets and algae. Mar. Ecol. Prog. Ser. 160:209-215.

Josselyn, M. N., and J. A. West. 1985. The distribution and temporal dynamics of the estuarine macroalgal community of San Francisco Bay. Hydrobiologia 129:139152.

Keough, M. J., and G. P. Quinn. 1998. Effects of periodic disturbances from trampling on rocky intertidal algal beds. Ecol. Appl. 8:141-161.

Kilar, J. A., and J. McLachlan. 1989. Effects of wave exposure on the community structure of a plant-dominated, fringingreef platform: Intermediate disturbance and disturbance-mediated competition. Mar. Ecol. Prog. Ser. 54:265-276.

Lewis, J. R. 1964. The ecology of rocky shores. The English Universities Press Ltd., London.

. 1968. Water movements and their role in rocky shore ecology. Sarsia 34:1336.

Littler, M. M. 1980a. Overview of the rocky intertidal systems of southern California. Pages 265-306 in D. M. Power, ed. The California Islands: Proceedings of a multidisciplinary symposium. Santa Barbara Museum of Natural History, Santa Barbara, California.

-1980b. Southern California rocky intertidal ecosystems: Methods, community structure and variability. Pages 565608 in J. H. Price, D. E. G. Irvine, and W. F. Farnham, eds. The shore environment. Vol. 2. Ecosystems. Academic Press, London.

Littler, M. M., and B. J. Kauker. 1984. Heterotrichy and survival strategies in the red 
alga Corallina officinalis. Bot. Mar. 27:3744.

Littler, M. M., and D. S. Littler. 1980. The evolution of thallus form and survival strategies in benthic marine macroalgae: Field and laboratory tests of a functional form model. Am. Nat. 116:25-44.

Littler, M. M., and S. N. Murray. 1975. Impact of sewage on the distribution, abundance and community structure of rocky intertidal macro-organisms. Mar. Biol. (Berl.) 30:277-291.

- 1978. Influence of domestic wastes on energetic pathways in rocky intertidal communities. J. Appl. Ecol. 15:583-595.

Littler, M. M., D. S. Littler, S. N. Murray, and R. R. Seapy. 1991. Southern California rocky intertidal ecosystems. Pages 273-296 in A. C. Mathieson and P. H. Nienhuis, eds. Ecosystems of the world. Vol. 24. Intertidal and littoral ecosystems. Elsevier, Amsterdam.

Littler, M. M., D. R. Martz, and D. S. Littler. 1983. Effects of recurrent sand deposition on rocky intertidal organisms: Importance of substrate heterogeneity in a fluctuating environment. Mar. Ecol. Prog. Ser. 11:129-139.

Lubchenco, J. 1980. Algal zonation in the New England rocky intertidal community: An experimental analysis. Ecology 61:333344.

1983. Littorina and Fucus: Effects of herbivores, substratum heterogeneity, and plant escapes during succession. Ecology 64:1116-1123.

Lubchenco, J., and B. A. Menge. 1978. Community development and persistence in a low rocky intertidal zone. Ecol. Monogr. 48:67-94.

Manly, B. F. J. 1997. Randomization and Monte Carlo methods in biology. 2nd ed. Chapman \& Hall, London.

Mann, K. H. 1982. Ecology of coastal waters: A systems approach. University of California Press, Berkeley.

Markham, J. W. 1973. Observations on the ecology of Laminaria sinclairii on three northern Oregon beaches. J. Phycol. 9:336-341.

Mathieson, A. C. 1979. Vertical distribution and longevity of subtidal seaweeds in northern New England, U.S.A. Bot. Mar. 30:511-520.

- 1989. Phenological patterns of northern New England seaweeds. Bot. Mar. 32:419-438.

Mathieson, A. C., C. A. Penniman, and L. G. Harris. 1991. Northwest Atlantic rocky shore ecology. Pages 109-191 in A. C. Mathieson and P. H. Nienhuis, eds. Ecosystems of the world. Vol. 24. Intertidal and littoral ecosystems. Elsevier, Amsterdam.

Mathieson, A. C., J. W. Shipman, J. R. O'Shea, and R. C. Hasevlat. 1976. Seasonal growth and reproduction of estuarine fucoid algae in New England. J. Exp. Mar. Biol. Ecol. 25:273-284.

McArdle, D. A. 1997. California marine protected areas. California Sea Grant College System, University of California, La Jolla, Publication No. T-039.

McQuaid, C. D., and G. M. Branch. 1985. Trophic structure of rocky intertidal communities: Response to wave action and implications for energy flow. Mar. Ecol. Prog. Ser. 22:153-161.

McQuaid, C. D., and K. M. Dower. 1990. Enhancement of habitat heterogeneity and species richness on rocky shores inundated by sand. Oecologia (Berl.) 84:142-144.

McQuaid, C. D., G. M. Branch, and A. A. Crowe. 1985. Biotic and abiotic influences on rocky intertidal biomass and richness in the Southern Benguela region. S. Afr. J. Zool. 20:115-122.

Menge, B. A. 1976. Organization of the New England rocky intertidal communities: Roles of predation, competition, and environmental heterogeneity. Ecol. Monogr. 46:355-393.

- 1991. Relative importance of recruitment and other causes of variation in rocky intertidal community structure. J. Exp. Mar. Biol. Ecol. 146:69-100.

Menge, J. L. 1975. Effect of herbivores on community structure of the New England rocky intertidal region: Distribution, abundance and diversity of algae. Ph.D. diss., Harvard University, Cambridge, Massachusetts.

Morris, R. H., D. P. Abbott, and E. C. Haderlie. 1980. Intertidal invertebrates 
of California. Stanford University Press, Stanford, California.

Munda, I. M., and J. W. Markham. 1982. Seasonal variations of vegetation patterns and biomass constituents in the rocky eulittoral of Helgoland. Helgol. Meeresunters. 35:131-151.

Murray, S. N. 1998. Effectiveness of California Marine Life Refuges on southern California shores. Pages 1453-1465 in O. T. Magoon, $\mathrm{H}$. Converse, B. Baird, and $M$. Miller-Henson, eds. California and the world ocean '97: Taking a look at California's ocean resources: An agenda for the future. American Society of Civil Engineers, Reston, Virginia.

Murray, S. N., and R. N. Bray. 1993. Benthic macrophytes. Pages 304-368 in M. D. Dailey, D. J. Reish, and J. W. Anderson, eds. Ecology of the Southern California Bight: A synthesis and interpretation. University of California Press, Berkeley.

Murray, S. N., and T. G. Denis. 1997. Vulnerability of the rockweed Pelvetia compressa to anthropogenic disturbance on southern California rocky shores. Phycologia 36 (4) Suppl.: 75-76.

Murray, S. N., and M. M. Littler. 1984. Analysis of seaweed communities in a disturbed rocky intertidal environment near Whites Point, Los Angeles, Calif., U.S.A. Hydrobiologia 116/117:374-382.

Murray, S. N., T. G. Denis, J. S. Kido, and J. R. Smith. 1999. Human visitation and the frequency and potential effects of collecting on rocky intertidal populations in southern California marine reserves. Calif. Coop. Oceanic Fish. Invest. Rep. 40:100106.

Niemeck, R. A., and A. C. Mathieson. 1976. An ecological study of Fucus spiralis. J. Exp. Mar. Biol. Ecol. 24:33-48.

Norton, T. A. 1986. The zonation of seaweeds on rocky shores. Pages 7-21 in P. G. Moore and R. Seed, eds. The ecology of rocky coasts. Columbia University Press, New York.

Norton, T. A., S. J. Hawkins, N. L. Manley, G. A. Williams, and D. C. Watson. 1990. Scraping a living: A review of littorinid grazing. Hydrobiologia 193:117-138.
Norton, T. A., A. C. Mathieson, and M. Neushul. 1982. A review of some aspects of form and function in seaweeds. Bot. Mar. 25:501-510.

Oates, B. R. 1981. Ecological and physiological studies concerning Hesperopbycus barveyanus (Decne.) S. \& G. and Pelvetia fastigiata f. gracilis S. \& G., two intertidal fucoids from Southern California. M.A. thesis, California State University, Fullerton.

Osenberg, C. W., and R. J. Schmitt. 1996. Detecting ecological impacts caused by human activities. Pages 3-16 in R. J. Schmitt and C. W. Osenberg, eds. Detecting ecological impacts: Concepts and applications in coastal habitats. Academic Press, New York.

Paine, R. T., and S. A. Levin. 1981. Intertidal landscapes: Disturbance and the dynamics of pattern. Ecol. Monogr. 51:145-178.

Pielou, E. C. 1981. Rapid estimation of the standing crop of intertidal fucoids on an exposed shore. J. Environ. Manage. 13:8598.

Pimentel, R. A., and J. D. Smith. 1990. Biostat I: A univariate statistical toolbox, version 2.0. Sigma Soft, Placentia, California.

Printz, H. 1950. Seasonal growth and production of dry matter in Ascopbyllum nodosum (L.) LeJol. Avh. Utgitt Nor. Vidensk. Akad. Oslo. I., Mar.-Naturvidensk. K. 4:115.

Pyefinch, K. A. 1943. The intertidal ecology of Bardsey Island, North Wales, with special reference to the recolonization of rock surfaces and the rock-pool environment. J. Anim. Ecol. 12:82-104.

Quinn, G. P., and M. J. Keough. 2002. Experimental design and data analysis for biologists. Cambridge University Press, Cambridge.

Raffaelli, D., and S. Hawkins. 1996. Intertidal ecology. Chapman \& Hall, London.

Ricketts, E. F., J. Calvin, J. W. Hedgpeth, and D. W. Phillips. 1985. Between Pacific tides. 5th ed. Stanford University Press, Stanford, California.

Schiel, D. R., and D. I. Taylor. 1999. Effects of trampling on a rocky intertidal algal 
assemblage in southern New Zealand. J. Exp. Mar. Biol. Ecol. 235:213-235.

Schonbeck, M., and T. A. Norton. 1978. Factors controlling the upper limits of fucoid algae on the shore. J. Exp. Mar. Biol. Ecol. 31:303-313.

1980. Factors controlling the lower limits of fucoid algae on the shore. J. Exp. Mar. Biol. Ecol. 43:131-150.

Seapy, R. R., and M. M. Littler. 1982. Population and species diversity fluctuations in a rocky intertidal community relative to severe aerial exposure and sediment burial. Mar. Biol. (Berl.) 71:87-96.

Shannon, C. E., and W. Weaver. 1949. The mathematical theory of communication. University of Illinois Press, Urbana.

Sideman, E. J., and A. C. Mathieson. 1983. The growth, reproductive phenology, and longevity of non-tide pool Fucus disticbus (L.) Powell in New England. J. Exp. Mar. Biol. Ecol. 72:171-188.

Smith, S. D. A., and R. D. Simpson. 1995. Effects of the 'Nella Dan' oil spill on the fauna of Durvillaea antarctica holdfasts. Mar. Ecol. Prog. Ser. 121:73-89.

Sousa, W. P. 1985. Disturbance and patch dynamics on rocky intertidal shores. Pages 101-124 in S. T. A. Pickett and P. S. White, eds. The ecology of natural disturbance and patch dynamics. Academic Press, New York.

Southward, A. J. 1956. The population balance between limpets and seaweeds on wave-beaten rocky shores. Rep. Mar. Biol. Stn. Port Erin. 68:20-29.

Stephenson, T. A. 1939. The constitution of the intertidal fauna and flora of South Africa-Part I. J. Linn. Soc. (Zool.) 40:487-536.

Stephenson, T. A., and A. Stephenson. 1972. Life between tidemarks on rocky shores. W. H. Freeman \& Co., San Francisco.

Stewart, J. G. 1982. Anchor species and epiphytes in intertidal algal turf. Pac. Sci. $36: 45-59$.

1983. Fluctuations in the quantity of sediments trapped among algal thalli on intertidal rocky platforms in southern California. J. Exp. Mar. Biol. Ecol. 73:205-211.
Stewart-Oaten, A., W. W. Murdoch, and K. R. Parker. 1986. Environmental impact assessment: "Pseudoreplication" in time? Ecology 67:929-940.

Thompson, R. C., B. J. Wilson, M. L. Tobin, A. S. Hill, and S. J. Hawkins. 1996. Biologically generated habitat provision and diversity of rocky shore organisms at a hierarchy of spatial scales. J. Exp. Mar. Biol. Ecol. 202:73-84.

Trowbridge, C. D. 1996. Demography and phenology of the intertidal green alga Codium setchelli: The enigma of local scarcity on sand-influenced rocky shores. Mar. Biol. (Berl.) 127:341-351.

Underwood, A. J. 1981. Structure of a rocky intertidal community in New South Wales: Patterns of vertical distribution and seasonal changes. J. Exp. Mar. Biol. Ecol. 51:57-85.

. 1997. Experiments in ecology. Cambridge University Press, Cambridge.

Underwood, A. J., and M. G. Chapman. 1996. Scales of spatial patterns of distribution of intertidal invertebrates. Oecologia (Berl.) 107:212-224.

Vadas, R. L., W. A. Wright, and S. L. Miller. 1990. Recruitment of Ascopbyllum nodosum: Wave action as a source of mortality. Mar. Ecol. Prog. Ser. 61:263-272.

Van Alstyne, K. L. 1990. Effects of wounding by the herbivorous snails Littorina sitkana and L. scutulata (Mollusca) on growth and reproduction of the intertidal algae Fucus distichus (Phaeophyta). J. Phycol. 26:412416.

Whorff, J. S., L. L. Whorff, and M. H. Sweet III. 1995. Spatial variation in an algal turf community with respect to substratum slope and wave height. J. Mar. Biol. Assoc. U.K. 75:429-444.

Williams, G. A. 1996. Seasonal variation in a low shore Fucus serratus (Fucales, Phaeophyta) population and its epiphytic fauna. Hydrobiologia 326/327:191-197.

Williams, S. L., and R. E. Di Fiori. 1996. Genetic diversity and structure in Pelvetia fastigiata (Phaeophyta: Fucales): Does a small effective neighborhood size explain fine-scale genetic structure? Mar. Biol. (Berl.) 126:371-382. 


\section{Appendix}

List of Species

\begin{tabular}{|c|c|}
\hline Taxa & Location $^{a}$ \\
\hline \multicolumn{2}{|l|}{ Division Chlorophyta } \\
\hline Chaetomorpba linum (Müll.) Kütz. & CRC, WDS, VIC, TRI, MBY, DPT \\
\hline Cladophoropsis fasciculatus (Kjellm.) Okam. & $\mathrm{DPT}$ \\
\hline Ulva californica Wille & CRC, SHW, WDS, VIC, TRI, THS, MBY \\
\hline \multicolumn{2}{|l|}{ Division Phaeophyta } \\
\hline Dictyota flabellata (Coll.) S. \& G. & CRC \\
\hline Halidrys dioica Gardn. & SHW \\
\hline Hapterophycus canaliculatus S. \& G. & SHW, WDS, TRI, THS, DPT \\
\hline Hesperopbycus californicus Silva & VIC, TRI, MBY \\
\hline Petrospongium rugosum (Okam.) S. \& G. & CRC, SHW, WDS, VIC \\
\hline Pseudolithoderma nigra Hollenb. & CRC, SHW, WDS, VIC, TRI, THS, MBY, DPT \\
\hline Ralfsia pacifica Hollenb. & CRC, WDS, MBY \\
\hline Sargassum muticum (Yendo) Fensh. & CRC, WDS \\
\hline Silvetia compressa (J. Ag.) Serrão, Cho, Boo \& Brawley & CRC, SHW, WDS, VIC, TRI, THS, MBY, DPT \\
\hline Zonaria farlowii S. \& G. & CRC \\
\hline \multicolumn{2}{|l|}{ Division Rhodophyta } \\
\hline Acrosorium venulosum ( $\mathrm{Zan}.) \mathrm{Kyl}$. & $\mathrm{DPT}$ \\
\hline Bossiella orbigniana (Dec.) Silva & TRI, MBY \\
\hline Callitbamnion rupicola Anders. & CRC, SHW, WDS, THS \\
\hline Centroceras clavulatum (C. Ag.) Mont. & $\mathrm{CRC}$ \\
\hline $\begin{array}{l}\text { Ceramium sinicola S. \& G. or Corallophila eatoniana } \\
\text { (Farl.) Cho, Choi, Hansen \& Boo }\end{array}$ & CRC, SHW, WDS, THS, MBY, DPT \\
\hline Cbondracantbus canaliculatus (Harv.) Guiry & CRC, SHW, WDS, THS, MBY, DPT \\
\hline Chondracanthus spinosus (Kütz.) Guiry & THS, DPT \\
\hline Chondria acrorbizophora S. \& G. & $\mathrm{DPT}$ \\
\hline Corallina pinnatifolia (Manza) Daws. & CRC, SHW, WDS, VIC, TRI, THS, MBY, DPT \\
\hline Corallina vancouveriensis Yendo & CRC, SHW, WDS, VIC, TRI, THS, MBY, DPT \\
\hline Cryptopleura crispa Kyl. & CRC, SHW, WDS, TRI, THS, MBY, DPT \\
\hline Gelidium coulteri Harv. & CRC, SHW, WDS, VIC, TRI, THS, MBY, DPT \\
\hline Gelidium pusillum (Stackh.) Le Jol. & CRC, SHW, WDS, VIC, TRI, THS, MBY, DPT \\
\hline Herposipbonia verticillata (Harv.) Kyl. & CRC, SHW, THS, MBY, DPT \\
\hline fania crassa Lamour. & CRC, MBY, DPT \\
\hline Fania tenella (Kütz.) Grun. & MBY \\
\hline Laurencia masonii S. \& G. & CRC, SHW, MBY, DPT \\
\hline Laurencia pacifica Kyl. & CRC, WDS, TRI, THS, MBY, DPT \\
\hline Laurencia spp. & CRC \\
\hline Litbotbrix aspergillum Gray & CRC, WDS, THS, MBY \\
\hline Mazzaella affinis (Harv.) Fredericq & CRC, TRI, THS, DPT \\
\hline Mazzaella leptorbynchos (J. Ag.) Leister & THS, DPT \\
\hline $\begin{array}{l}\text { Osmundea crispa (Hollenb.) Nam and O. sinicola (S. \& G.) } \\
\text { Nam }\end{array}$ & WDS, MBY \\
\hline Peyssonneliaceae/Hildenbrandaceae & CRC, SHW, WDS, VIC, TRI, THS, MBY, DPT \\
\hline Plocamium cartilaginueum (L.) Dix. & CRC, THS, MBY \\
\hline Polysiphonia pacifica Hollenb. & CRC, SHW, THS \\
\hline Polysiphonia scopulomem Harv. & CRC, MBY \\
\hline Polysipbonia spp. & CRC, THS \\
\hline Pseudolithopbyllum neofarlowii (Setch. \& Mason) Adey & CRC, SHW, WDS, VIC, TRI, THS, MBY, DPT \\
\hline Pterocladiella capillacea (Gmel.) Santelices \& Hommersand & CRC, SHW, TRI, THS, MBY, DPT \\
\hline Pterosipbonia baileyi (Harv.) Falk. & THS \\
\hline Pterosipbonia dendroidea (Mont.) Falk. & CRC, SHW, WDS, THS, MBY, DPT \\
\hline Unidentified crustose coralline & CRC, SHW, WDS, TRI, THS, MBY, DPT \\
\hline Unidentified Gigartinales & CRC, WDS, MBY \\
\hline \multicolumn{2}{|l|}{ Phylum Porifera } \\
\hline Unidentified sponge & CRC, SHW, WDS, VIC, TRI, MBY \\
\hline \multicolumn{2}{|l|}{ Phylum Cridaria } \\
\hline Anthopleura elegantissima (Brandt, 1835) & CRC, SHW, WDS, VIC, TRI, THS, MBY, DPT \\
\hline
\end{tabular}


Appendix (continued)

\begin{tabular}{|c|c|}
\hline Taxa & Location $^{\prime}$ \\
\hline \multicolumn{2}{|l|}{ Phylum Annelida } \\
\hline Pbragmatopoma califormica (Fewkes, 1889) & CRC, SHW, WDS, VIC, TRI, THS, MBY, DPT \\
\hline Spirobranchus spinosus Moore, 1923 & CRC, SHW, WDS, TRT, MBY \\
\hline \multicolumn{2}{|l|}{ Phylum Arthropoda } \\
\hline Balanus glandula Darwin, 1854 & CRC, SHW, WDS, VIC, TRI, THS, DPT \\
\hline Chtbamalus dalli Pilsbry, 1916 & CRC, SHW, WDS, VIC, TRI, THS, MBY, DPT \\
\hline Cbtbamalus fissus Darwin, 1854 & CRC, SHW, WDS, VIC, TRI, THS, MBY, DPT \\
\hline Pacbygrapsus crassipes Randall, 1839 & CRC, SHW, WDS, VIC, TRI, THS, MBY, DPT \\
\hline Pagurus birsutiusculus venturensis Coffin, 1957 & SHW, WDS, VIC, TRI, MBY, DPT \\
\hline Pagurzes samuelis (Stimpson, 1857) & CRC, SHW, WDS, TRI, THS, MBY, DPT \\
\hline Pollicipes polymerus Sowerby, 1833 & CRC, SHW, WDS, VIC, TRI \\
\hline Pugettia producta (Randall, 1839) & $\mathrm{SHW}, \mathrm{MBY}$ \\
\hline Tetraclita rubescens Darwin, 1854 & CRC, SHW, WDS, VIC, TRI, MBY, DPT \\
\hline \multicolumn{2}{|l|}{ Phylum Mollusca } \\
\hline Acantbina punctulata (Sowerby, 1825) & TRI, MBY, DPT \\
\hline Acantbina spirata (de Blainville, 1832) & CRC, SHW, WDS, VIC, TRI, THS, MBY, DPT \\
\hline Amphissa versicolor Dall, 1871 & TRI \\
\hline Bittium attenuatum (Carpenter, 1864) & TRI, MBY \\
\hline Bracbidontes adamsianus (Dunker, 1857) & CRC, SHW, WDS, VIC, TRI, THS, MBY, DPT \\
\hline Ceratostoma nuttalli (Conrad, 1837) & MBY \\
\hline Conus californicus Reeve, 1844 & CRC, SHW, THS, MBY, DPT \\
\hline Crepidula adunca Sowerby, 1825 & TRI, MBY \\
\hline Dendropona lituella (Mörch, 1861) & CRC, WDS \\
\hline Diaulula sandiegensis (Cooper, 1862) & MBY \\
\hline Epitonium tinctum (Carpenter, 1864) & CRC, SHW, WDS, VIC, TRI, THS, MBY, DPT \\
\hline Fissurella volcano Reeve, 1849 & CRC, SHW, WDS, MBY, DPT \\
\hline Glans subquadrata (Carpenter, 1864) & MBY \\
\hline Hipponex tumens Carpenter, 1864 & WDS, MBY \\
\hline Lepidochitona dentiens (Gould, 1846) & VIC \\
\hline Lepidocbitona bartwegii (Carpenter, 1855) & CRC, SHW, WDS, VIC, TRI, THS, MBY, DPT \\
\hline Lithopbaga phumula (Hanley, 1843) & MBY \\
\hline Littorina keenae Rosewater, 1978 & SHW, WDS, VIC, TRI, THS, MBY, DPT \\
\hline Littorina scutulata Gould, 1849 & CRC, SHW, WDS, VIC, TRI, THS, MBY, DPT \\
\hline Lottia asmi (Middendorff, 1847) & WDS, DPT \\
\hline Lottia conus (Test, 1945) & CRC, SHW, WDS, VIC, THS, DPT \\
\hline Lottia digitalis (Rathke, 1833) & WDS, TRI, MBY \\
\hline Lottia gigantea Sowerby, 1834 & SHW, WDS, VIC, TRI \\
\hline Lottia limatula (Carpenter, 1864) & CRC, SHW, WDS, VIC, TRI, THS, MBY, DPT \\
\hline Lottia pelta (Rathke, 1833) & CRC, SHW, WDS, VIC, TRI, THS, MBY, DPT \\
\hline Lottia spp. & SHW, WDS, VIC, MBY \\
\hline Lottia strigatella (Carpenter, 1864) & CRC, SHW, WDS, VIC, TRI, THS, MBY, DPT \\
\hline Macclintockia scabra (Gould, 1846) & CRC, SHW, WDS, VIC, TRI, THS, MBY, DPT \\
\hline Macron lividus (A. Adams, 1855) & CRC, SHW, WDS, TRI \\
\hline Maxwellia gennna (Sowerby, 1879) & $M B Y$ \\
\hline Mopalia ciliata (Sowerby, 1840) & TRI \\
\hline Mopalia muscosa (Gould, 1846) & CRC, SHW, WDS, VIC, TRI, THS, MBY, DPT \\
\hline Mytilus californianus Conrad, 1837 & CRC, SHW, WDS, VIC, TRI, THS, MBY, DPT \\
\hline Mytilus galloprovincialis L., 1758 & CRC, SHW, WDS \\
\hline Norrisia norrisi (Sowerby, 1838) & $\mathrm{CRC}, \mathrm{MBY}$ \\
\hline Nucella emarginata (Deshayes, 1839) & CRC, SHW, WDS, VIC, TRI, THS, DPT \\
\hline Nucella lamellosa Gmelin, 1791 & VIC \\
\hline Nucella spp. & VIC \\
\hline Nuttallina californica (Reeve, 1847) & SHW, WDS, VIC, TRI, THS, MBY, DPT \\
\hline Nuttallina fluxa (Carpenter, 1864) & CRC, SHW, WDS, VIC, TRI, THS, MBY, DPT \\
\hline Petaloconcbus montereyensis Dall, 1919 & CRC, SHW, WDS, TRI, DPT \\
\hline Pseudochama exogyra (Conrad, 1837) & CRC, SHW, WDS, VIC, TRI, THS, MBY, DPT \\
\hline Septifer bifurcatzis (Conrad, 1837) & CRC, SHW, WDS, VIC, TRI, THS, MBY, DPT \\
\hline
\end{tabular}


Appendix (continued)

\begin{tabular}{ll}
\hline \hline Taxa & \multicolumn{1}{c}{ Location $^{\prime}$} \\
\hline Serpulorbis squamigerzis (Carpenter, 1857) & CRC, SHW, WDS, TRI, MBY \\
Stenoplax conspicua Pilsbry, 1892 & THS \\
Tegula autreotincta (Forbes, 1852) & SHW, MBY, DPT \\
Tegula eiseni Jordan, 1936 & CRC, SHW, TRI, THS, MBY, DPT \\
Tegula funebralis (A. Adams, 1855) & CRC, SHW, WDS, VIC, THS, MBY, DPT \\
Tegula gallina (Forbes, 1852) & CRC, SHW, THS, MBY, DPT \\
Phylum Echinodermata & \\
Strongylocentrotus purpuratus (Stimpson, 1857) & MBY \\
Phylum Ectoprocta & \\
Unidentified bryozoan & CRC, SHW, WDS, VIC, TRI, MBY, DPT \\
\hline
\end{tabular}

"See Figure 1 for explanation. 